\title{
A neural circuit basis for bilateral olfactory input-enhanced chemosensory avoidance navigation
}

Samuel K. H. Sy, ${ }^{1,2,10-12}$ Danny C. W. Chan, ${ }^{1-4}$ Hei-Ming Lai, ${ }^{1,25,6,7}$ Zhongqi Li, ${ }^{1,2}$ Kenneth K. Y. Wong, ${ }^{11,12}$ Chung Hang Jonathan Choi, ${ }^{8,10}$ Vincent C. T. Mok, ${ }^{1,6-8} \mathrm{Yu} \mathrm{Hu},{ }^{13} \mathrm{Ho} \mathrm{Ko}{ }^{1,2,4-9,14, *}$

\section{Affiliations}

${ }^{1}$ Division of Neurology, Department of Medicine and Therapeutics, ${ }^{2} \mathrm{Li}$ Ka Shing Institute of Health Sciences, ${ }^{3}$ Department of Anaesthesia and Intensive Care, ${ }^{4}$ Peter Hung Pain Research Institute, ${ }^{5}$ Department of Psychiatry, ${ }^{6}$ Margaret K. L. Cheung Research Centre for Management of Parkinsonism, ${ }^{7}$ Gerald Choa Neuroscience Centre, ${ }^{8}$ Chow Yuk Ho Technology Centre for Innovative Medicine, ${ }^{9}$ School of Biomedical Sciences, Faculty of Medicine, ${ }^{10}$ Department of Biomedical Engineering, Faculty of Engineering, The Chinese University of Hong Kong.

${ }^{11}$ Department of Electrical and Electronic Engineering, Faculty of Engineering, The University of Hong Kong.

${ }^{12}$ Advanced Biomedical Instrumentation Centre, Hong Kong Science Park, Shatin, New Territories, Hong Kong.

${ }^{13}$ Department of Mathematics and Division of Life Science, Faculty of Science, Hong Kong University of Science and Technology.

${ }^{14}$ Lead Contact.

*Correspondence: ho.ko@cuhk.edu.hk (H.K.)

\section{Summary}

Optimizing navigational strategies in nature requires the detection and processing of survival-relevant cues. Our understanding of how animals utilise parallel inputs from paired sensory organs for this purpose and the underlying neural circuit mechanisms remain limited. Here we developed microfluidics-based behavioral and brainwide imaging platforms to study the neural integration of bilateral olfactory inputs and chemosensory avoidance in larval zebrafish. We show that larval zebrafish efficiently escape from cadaverine-carrying streams using undirected large turns, where both angular velocity and adaptation of turn duration exhibit bilateral olfactory input-dependence. In contrast, concomitant swim bout frequency modulation (i.e., klinokinesis) only requires unilateral input. Throughout the olfactory processing pathways, a distributed neural representation with a wide spectrum of ipsilateral-contralateral stimulus selectivity is maintained. Nonlinear sensory information gain with bilateral signal convergence is especially prominent in neurons weakly encoding unilateral cadaverine stimulus, and associated with stronger activation of sensorimotor neurons in the downstream brain regions. Collectively, these results provide insights into how the vertebrate model sums parallel input signals to guide navigational behavior. 


\section{Introduction}

Bilaterian animals have paired sensory organs with symmetrical anatomical locations. Information extraction by integrating paired sensory organ inputs across different sensory modalities have been studied in numerous species including humans (Mills, 1958; Porter et al., 2006; Westheimer, 1990), mammals (Brand et al., 2002; Chioma et al., 2020; Heffner, 2004; Heffner and Heffner, 1988; Ohzawa et al., 1996) and insects (Martin, 1965; Steck et al., 2010). In principle, such design offers several potential advantages that can be exploited. Depending on the specific circumstances, inputs from spatially segregated, parallel sensory channels may be summed (or averaged) to enhance the signal-to-noise ratio of cue detection, or used in stereo comparison for spatial information extraction. Classical examples include the utilization of interaural level and timing differences for sound source localization (Schnupp and Carr, 2009), and visual binocular disparity for depth perception (Qian, 1997).

For chemotaxis and olfactory-mediated behaviors, the role of bilateral sensory input appears to be species- and context-dependent. Humans are capable of using inter-nostril comparison to enhance scent tracking (Porter et al., 2006). Rodents employ a strategy combining sequential sniffing and stereo comparison to efficiently localize odorant sources based on internasal odor intensity and arrival time differences (Catania, 2013; Liu et al., 2020; Rajan et al., 2006). In Drosophila larvae, bilateral dorsal olfactory organ inputs improve odor gradient detection during serial sampling to guide navigation (Louis et al., 2008). Adult Drosophila instead appear capable of stereo comparison between olfactory antenna inputs for odorant localization (Gomez-Marin et al., 2010). Similarly, a dependence of chemotaxis on bilateral olfactory inputs has been reported in some insects, such as honey bees and ants (Martin, 1965; Steck et al., 2010). For aquatic animals, sampling chemical cues carried by water flow that signify danger or food source is essential for survival. Despite well-known chemosensory avoidance and attraction behaviors in various aquatic species (Chivers and Smith, 2016; Koide et al., 2009; Krishnan et al., 2014; Lee and Meyers, 1996; Wagner et al., 2011; Wakisaka et al., 2017), how bilateral olfactory inputs are utilised in guiding these behaviors remains unknown.

Moreover, the neural basis of processing parallel inputs from paired sensory organs for the instruction of subsequent motor output in vertebrates, are also incompletely understood. While interhemispheric projection pathways arising from early sensory pathways or higher-order brain areas are well-described in many species (Oh et al., 2014; Suárez et al., 2018), detailed circuit mechanisms of how these pathways mediate sensory processing are only beginning to be unraveled (Gebhardt et al., 2019; Hagihara et al., 2020; Kermen et al., 2020; Longordo et al., 2013; Rock and Apicella, 2015). Limited by tools available for studying the relatively large brains of many species, past works mostly focused on individual regions, such as the binocular visual areas (Hubel and Wiesel, 1962; Kara and Boyd, 2009; Longordo et al., 2013; Parker, 2007), the primary auditory cortex (Brugge et al., 1969; Imig and Adrián, 1977; Reser et al., 2000; Schnupp and Carr, 2009; Schnupp et al., 2001; Zhang et al., 2004) or restricted areas of the olfactory processing pathways (Kikuta et al., 2008; Yan et 
al., 2008). However, both the integration of bilateral sensory input and subsequent sensorimotor transformation may occur on a distributed, brainwide scale. Near-complete sampling of whole-brain neuronal dynamics during sensory processing and behavior is therefore a powerful approach to gain more detailed understanding of the underlying circuit mechanisms (Loring et al., 2020; Mu et al., 2020; Vanwalleghem et al., 2018).

The zebrafish olfactory system shares a conserved general circuit layout as other vertebrates, and offers the distinct advantage of permitting optical imaging of relatively large proportions of neurons along the early olfactory pathways (Friedrich, 2013). It is thus an attractive model system for studying the computational principles of sensory processing (Friedrich, 2013). Remarkably, within one-week post-fertilization, larval zebrafish already have a rich repertoire of innate behaviors. These include chemosensory behaviors, alongside phototaxis, oculomotor and optokinetic responses, prey capture and various escape responses (Kalueff et al., 2013; Loring et al., 2020). With a relatively small, transparent brain that comprises a more tractable number of neurons $\left(\sim 10^{5}\right)$, much has been learnt about the circuitries mediating sensory processing and sensorimotor behaviors in larval zebrafish (Ahrens et al., 2012; Bahl and Engert, 2020; Chen et al., 2018; Dragomir et al., 2020; Fernandes et al., 2020; Gebhardt et al., 2019; Haesemeyer et al., 2018; Herrera et al., 2020; Kawashima et al., 2016; Knogler et al., 2017; Lacoste et al., 2015; Lovett-Barron et al., 2020; Migault et al., 2018; Naumann et al., 2016; Portugues et al., 2014; Vanwalleghem et al., 2020; Wee et al., 2019; Wolf et al., 2017; Yao et al., 2016; Zhang et al., 2017). Larval zebrafish also offers a unique opportunity for studying the brainwide organizational principles of neural circuits mediating bilateral sensory input integration. For example, circuit processing principles for the binocular visual input-driven optomotor response (OMR) (Naumann et al., 2016) and prey capture (Gebhardt et al., 2019) behaviors have been studied.

In this work, we use larval zebrafish as the model organism to study the neural basis of parallel olfactory input integration in chemosensory avoidance. We first develop a laminar flow-based navigation assay with a static chemical border, to investigate the escape behavior of larval zebrafish upon naturalistic encounters of aversive stimulus. Using an odorant (cadaverine) which does not elicit strong escape responses yet is survival-relevant to avoid, we demonstrate navigation behavioral changes with significant consequences on escape efficiency that differentially depend on the integrity of bilateral olfactory placodes (OPs). We also develop an integrated microfluidics-light sheet imaging system to interrogate whole-brain neuronal dynamics during spatiotemporally precise unilateral or bilateral olfactory stimulation in restrained larval subjects. This approach enables us to reveal a distributed neural representation of cadaverine sensing, characterized by a wide range of ipsilateral-contralateral input selectivity throughout brain regions along the rostral-caudal axis, and the convergence of bilateral afferent signals on a brainwide scale. We show that sensory information gain with bilateral signal convergence is generally supralinear, and such synergistic input summation is associated with stronger activation of sensorimotor and motor output-encoding neuronal subsets. 


\section{Results}

\section{Larval zebrafish requires bilateral olfactory inputs to efficiently escape cadaverine zone}

In the natural environment, a fish needs to navigate away from zones with noxious chemicals. To study chemosensory avoidance in navigational behavior under a mimicking condition, we developed a microfluidics-based setup that maintains a precisely defined spatio-chemical landscape. The setup employs a rectangular swimming arena with three fluid inlets and two fluid outlets (Figure 1A). Computational fluid dynamics simulation shows laminar flow at steady state with very low flow velocity profile over the majority of the area except near inlets and outlets (Supplementary Figure 1A). The laminar flow enables the maintenance of static boundaries between fluidic stream zones with different chemical compositions (Figure 1A, Supplementary Figure 1B, see Methods). During an avoidance navigation assay, three to six zebrafish larvae aged 5-7 days post-fertilization (d.p.f.) freely swim in the arena with a noxious chemical zone in the absence of visible light (Figure 1A). We carried out high-speed imaging ( $240 \mathrm{~Hz}$ frame rate) under infrared (IR) illumination and tracking of larval zebrafish head orientation and coordinates for the quantification of navigational behavior (Figure 1A, see Methods). With a static chemical zone boundary, this assay allowed us to repeatedly sample events of entering and escaping the well-defined noxious chemical zone. Assays with water streams in all zones served as control.

Cadaverine is a diamine compound formed from the putrefaction of corpses. Apart from being an alarm cue, we observed that it is survival-relevant for larval zebrafish to avoid, as persistent exposure to cadaverine $(1 \mathrm{mM})$ in a survival assay invariably led to death (Figure 1B, see Methods). However, simply inspecting individual events of encountering cadaverine during the navigation behavioral assay, the larvae appeared incapable of performing immediate, strong escape, in line with a previous report (Koide et al., 2018). Yet, during more prolonged assay ( 2 hours), the zebrafish larvae exhibited clear avoidance of the cadaverine zone, as they spent substantially less time in the cadaverine zone than the spatially identical stimulus zone filled by water stream (control zone) (Figure 1C). This suggested that cadaverine avoidance in the larval zebrafish arises from more subtle navigation behavioral changes, that requires sufficient observations to reveal. To test the dependence of the avoidance behavior on the integrity of bilateral olfactory input, we performed two-photon laser ablation of unilateral olfactory placode (OP) and carried out the assay in two additional groups of larvae (i.e., either the left or the right OP was ablated, Figure 1D). Interestingly, although the unilateral OP-intact (uOP) larvae could still avoid the cadaverine zone, the proportions of time spent in the cadaverine zone were higher than the bilateral OP-intact (bOP) larvae (Figure 1D).

To compare escape efficiency from the noxious odor zone, we examined several metrics of navigational performance, including number of swim bouts (as zebrafish larvae swim in discrete bouts), time and distance travelled to escape for each cadaverine zone entry event (Figure 1E). The bOP larvae escaped from the cadaverine zone with fewer bouts than the uOP larvae (Figure 1F), and could escape using only one or two bouts in over $60 \%$ of the 
events, while the corresponding proportions for the uOP larvae in cadaverine zone were only slightly higher than that of the bOP larvae in control zone (Figure 1G). Consequently, the bOP larvae on average travelled significantly shorter distances (Figure 1H) and took less time (Figure 1I) to leave the cadaverine zone than the uOP larvae. In the larval zebrafish, the performance of chemosensory avoidance navigation therefore depends on the integrity of bilateral olfactory inputs.

\section{Bilateral olfactory input modulates turning behavior during avoidance navigation}

Several navigational strategies that utilise different combinations of swim or turn kinematic changes could in theory account for the observed bilateral olfactory input-dependent difference in cadaverine avoidance efficiency. These include increase in swim bout frequency (klinokinesis), angular or linear velocity (angular / linear orthokinesis) (Supplementary Figure 2A). To understand the strategy employed by larval zebrafish and identify any dependence on bilateral olfactory input, we next analysed swim bout kinematic parameters for the different groups in control and cadaverine zones. During the assay, occasionally the larvae came into contact with each other or the arena boundary and elicited touch-initiated responses, or performed mechanosensory-based rheotaxis near the fluid inlets and outlets where fluid velocities were higher (Supplementary Figure 1A). As we focused on chemical-induced behaviors, the trajectories of these events were excluded from analysis on swim bout kinematics (see Methods).

Prompted by the observation that the bOP larvae could escape within two swim bouts at a greater frequency than the uOP larvae (Figure 1G), we first focused on the initial swim bouts after encountering cadaverine (Figures 2A and 2B). Comparing the perpendicular displacements from the zone border of first turns for each group, only bOP larvae exhibited a tendency towards navigating away from the noxious odor zone (Figure 2C). The differential outcomes could be explained by larger turns the bOP larvae made during the first swim bouts in the cadaverine zone compared to the control zone, with a median angular magnitude difference approximately twice as large as that of the uOP larvae (Figures 2D and 2E). The angular magnitude difference was due to a higher angular velocity of the turns by the bOP larvae (Figure 2F), but not longer bout durations (Figure 2G). Note the highly overlapping distributions of the kinematic parameters, indicating a behavioral change that manifests only statistically as biases that nevertheless significantly impacts navigational performance.

Interestingly, we observed bilateral input-dependent adaptation of swim bout angular magnitude upon persistent cadaverine exposure. Although both bOP and uOP larvae maintained higher angular magnitude in the cadaverine zone than bOP larvae in the control zone on subsequent swim bouts (Figure 3A), the angular magnitude of bouts bOP larvae made converged onto that of $\mathrm{UOP}$ larvae with increasing bout numbers in the cadaverine zone (Figure 3B). This adaptation phenomenon was only evident in bOP larvae (Figure 3B), and occurred as a consequence of decreasing durations of subsequent turns (Figure 3C), despite the persisted elevation of angular velocity (Figure 3D). 
Additionally, both bOP and uOP larvae exhibited increased swim bout frequency in the cadaverine zone compared to the control zone (Figure 3E). This klinokinetic behavior only required unilateral olfactory input, as there were no significant differences in swim bout frequency between the bOP and uOP larvae in the cadaverine zone (Figure 3E). On the other hand, only minimal differences in swim bout linear velocity in the noxious odor zone was observed between the groups (Supplementary Figures 2B and 2C). If larval zebrafish were capable of stereo comparison and lateralization of odor, the bOP larvae should be able to make directional turns depending on odor zone border-crossing angle, while the uOP larvae should exhibit turn bias towards the ablated side upon encountering noxious odor. However, neither phenomenon was present (Figure 3A, Supplementary Figures 2D and 2E).

Collectively, we thus concluded that under the assay, larval zebrafish relies on bilateral olfactory inputs to drive faster and larger undirected turns more frequently, for efficient escape from the noxious cadaverine zone (i.e., klinokinesis in addition to angular orthokinesis). Klinokinesis only requires unilateral olfactory input, while angular orthokinesis is roughly halved with unilateral olfactory input, and exhibits adaptation whereby turn duration decreases when persistent odor signals are bilaterally sampled.

\section{Whole-brain neuronal imaging with spatiotemporally precise olfactory stimulation}

Studying bilateral olfactory input integration in larval zebrafish poses a unique methodological challenge due to the spatial proximity of the OPs $(\sim 100 \mu \mathrm{m}$ apart $)$. We developed a poly(dimethylsiloxane) (PDMS)-based microfluidic device for partially restraining a larval zebrafish and directed chemical delivery to one or both OPs, that is fully compatible with simultaneous whole-brain imaging by light sheet fluorescence microscopy (Ahrens et al., 2013; Keller et al., 2008; Panier et al., 2013) (Figure 4). The major functional compartments of the device include sets of chemical delivery microchannels, a head and waist-trapping chamber with a vertical and smooth side wall to ensure undisrupted propagation of incident excitation laser sheet (Figure 4A and Supplementary Figure 3A, also see Methods), and a tail chamber under IR illumination for the recording of tail flicking behavior at $200 \mathrm{~Hz}$ (Figure 4B). To overcome unwanted chemical spillover (Supplementary Figure 3B), we adopted a design whereby valve-gated fluid streams delivered from each side contain a central chemical stream which is insulated from the OP by the posterior water stream (Figure 4C, Supplementary Figures 3B and 3C, left). Spatiotemporally precise odor stimulus is delivered by turning off the insulating water stream(s), thereby allowing the chemical stream(s) to contact the respective $\mathrm{OP}(\mathrm{s})$, and terminated by turning off the chemical stream(s) (Figure 4C, Supplementary Figure 3C, middle and right). The stimulus profile visualized by a low concentration of sodium fluorescein $(1 \mu \mathrm{M})$ was characterized by a rapid rise reaching a plateau (mimicking the encounter of a chemical in a fluid stream), followed by a fast decay phase (see example traces in Figure 4C, also see Methods).

Using our integrated microfluidics-light sheet imaging system, we performed volumetric whole-brain calcium imaging in 5-6 d.p.f. larval zebrafish expressing the calcium reporter GCaMP6f in neurons (Chen et al., 2013), spanning 29 planes with 7- $\mu$ m intervals at 
$2 \mathrm{~Hz}$ (Figure 4D), while cadaverine $(1 \mathrm{mM})$ was presented to unilateral or bilateral OPs. A custom code-based workflow incorporating anatomical registration to the Z-Brain Atlas (Randlett et al., 2015), time-series image registration (Pnevmatikakis and Giovannucci, 2017), and calcium signal extraction (Giovannucci et al., 2019) was developed for integrated data analysis (Figure 4E, see Methods). We performed imaging in two configurations that permitted the incoming excitation light sheet to illuminate the majority of the whole brain, including the forebrain regions between the eyes (Supplementary Figure 4A): (1) with surgical ablation of the right eye of the larvae 12-24 hours prior to imaging (Supplementary Figure 4B), or (2) tilting the larvae by up to $20^{\circ}$ to the right (Supplementary Figure 4C). Both approaches circumvented the occlusion of the forebrain from imaging (Supplementary Figures 4D-F). Trials of different stimulus conditions (null, left OP stimulation (1-STIM), right OP stimulation (r-STIM), or bilateral OP stimulation (b-STIM)) were presented in a randomized order.

We carried out experiments in 18 right eye-ablated and 6 tilted larvae with at least 3 occurrences of each stimulus condition. Among the right eye-ablated larvae, $50 \%$ ( 9 out of 18) exhibited tail flicking in the device, while none of the tilted larvae were behaviorally responsive. Comparing the tail flick events of the behaviorally responsive larvae across the stimulus conditions, we noted tendencies of higher tail flick frequency and angular velocity during bilateral cadaverine stimulation than control (i.e., trials with no stimulation) or unilateral stimulation (Supplementary Figure 5). Compounded by the feasible number of repetitions, the stochastic nature of response to cadaverine, and possibly physical restraint and behavioral passivity of the larvae (Andalman et al., 2019; Mu et al., 2019), there were no statistically significant differences in these parameters across the conditions (Supplementary Figure 5). Although the larvae were not as responsive as in the swimming arena, the system permitted us to study brainwide olfactory sensory representation, and identify the regional distribution of neurons that encode sensory stimulation-associated motor output.

\section{Brainwide neuronal activation patterns with olfactory input and motor output}

Along the early olfactory pathway of larval zebrafish, olfactory bulb (OB) neurons project to bilateral OBs and diverse forebrain regions (Kunst et al., 2019; Miyasaka et al., 2014), but it is unclear whether the ipsilateral or the contralateral pathway is functionally dominant. Further down the pathways, it is also not known how unilateral or bilateral olfactory input determines the propagation of neuronal activity and sensory representation.

We first analyzed neuronal activity evoked by cadaverine under each stimulus condition. We identified sensory-encoding neurons, defined as those with the most significant mutual information $\left(I_{\mathrm{S}}\right)$ between activity and olfactory cadaverine stimulus, with 1-STIM, r-STIM or b-STIM (see Methods), from 9 stably imaged larvae ( $n=6$ behaviorally responsive, right eye-ablated and 3 tilted-imaged larvae with sufficiently stable recordings for high-quality across-trial registrations). Unexpectedly, in 5 out of 6 stably imaged right eye-ablated and behaviorally responsive larvae, r-STIM resulted in stronger activation than 1-STIM in the majority of brain regions (except left olfactory epithelium (OE), see 
Supplementary Figures 6A and 6B). This phenomenon was potentially due to altered cross-modality sensory response modulation after right eye ablation, and was not observed for the remaining right eye-ablated larva and 3 tilted-imaged larvae (Figures $5 \mathbf{A}$ and 5B, Supplementary Figures 6C and 6D). We separated our analyses for animals exhibiting unbiased vs. biased neural responses to r-STIM (see Methods for classification). For unbiased animals $\left(n=4\right.$ larvae), $I_{\mathrm{S}}$ of individual neurons were directly further analyzed (Figures 5A and 5B, Supplementary Figures 6C and 6D). In a separate analysis, $I_{\mathrm{S}}$ of individual neurons from each animal were normalized to the mean $I_{\mathrm{S}}$ of its left, right or bilateral OBs during 1-STIM, r-STIM or b-STIM, respectively $\left(I_{\mathrm{N}}\right)$, to permit pooling and visualization across all larvae $(n=9)$ (Figures 5C and 5D).

We then examined whole-brain neuronal activity patterns (Figures 5A-D, Supplementary Figures 6C and 6D). Unilateral OP stimulation results in strong activation of olfactory sensory neurons (OSNs) in the ipsilateral OE. Surprisingly, very weak activation in the contralateral $\mathrm{OE}$ was also noted, even though we were certain there was no cadaverine spillover (see Methods). Along the olfactory pathway, we found robust bilateral neuronal activation as early as in the OBs. In the forebrain, neurons in the bilateral pallium (Pa, or dorsal telencephalon) and subpallium ( $\mathrm{sPa}$, or ventral telencephalon) both respond to unilateral olfactory stimulus. Although a known asymmetrical bulbo-habenular pathway projects selectively to the right habenula ( $\mathrm{Hb}$ ) (Miyasaka et al., 2009), in the majority of the larvae the left $\mathrm{Hb}$ neurons were similarly activated as the right counterpart. The preoptic area (PO), a hypothalamic region known to mediate nocifensive behaviors (Wee et al., 2019), also generated bilateral neuronal activity during unilateral OP stimulation. There is a progressively decreasing trend of sensory encoding along the rostral-caudal axis in these regions. Beyond the forebrain regions, there is only direct sensory encoding by small subsets of neurons in the diencephalon (Di), mesencephalon (Me), cerebellum (Ce) and rhombencephalon (Rh). Notably, during bilateral OP stimulation, symmetrical regional activation with an increase in the sensory information contents of individual neurons' responses was observed. This points to a bilateral input-dependent propagation of sensory-evoked neuronal activities.

From the behaviorally responsive larval zebrafish, we also calculated the mutual information between individual neuronal activity and tail flick movements recorded $\left(I_{\mathrm{M}}\right)$ (see Methods). The motor-encoding neurons were mostly found in the more caudal brain regions where less neurons are direct sensory-encoding (i.e., Di, Me and Rh), and only very few were found in the forebrain regions (Figures $\mathbf{5 E}-\mathbf{H}$ ). Collectively, we were thus able to reveal the brainwide activation patterns underlying unilateral or bilateral olfactory sensory input processing, as well as the regional distributions of motor output-encoding units.

\section{Bilateral input integration in the early olfactory pathway and higher brain regions for cadaverine sensing}

We then investigated the rules of olfactory sensory input integration for cadaverine sensing. Firstly, we characterized the propagation of information evoked from unilateral 
stimulation in terms of neuronal and regional selectivity to ipsilateral vs. contralateral sources. For the larvae with unbiased responses to unilateral stimulation $(n=4)$, we calculated the ipsilateral input selectivity of each neuron (defined as $I_{\mathrm{S}_{-} \text {ipsi-STIM }} /\left(I_{\mathrm{S}_{\text {_ipsi-STIM }}}+\right.$ $I_{\text {S_contra-STIM }}$, where $I_{\text {S_ipsi-STIM }}$ and $I_{\text {S_contra-STIM }}$ denote mutual information between calcium activity and ipsilateral or contralateral OP stimulus, respectively). In all regions except the OE, neurons fall within a wide distribution of input preference, ranging from being highly ipsilaterally selective to highly contralaterally selective (Figures 6A and 6B). In the OBs, where subsets of neurons respond to both ipsilateral and contralateral OP cadaverine stimulation, the overall higher ipsilateral selectivity inherited from the OE remains partially conserved (Figures 6A-D). The ipsilateral input bias becomes weaker in the $\mathrm{Pa}$ and the sPa, as well as in the other more caudal brain regions (Figures 6A-C). Therefore, while within each region a wide range of response selectivity is maintained, bilateral olfactory input signals converge both early in the olfactory processing pathway and in the downstream regions. This indicates a brainwide, distributed mode of parallel input integration.

To examine the linearity of bilateral input integration, we next fitted generalized linear models (GLMs) to the responses of sensory-encoding neurons (Supplementary Figure 7) for all larvae $(n=9)$. We compared GLMs with kernels for 1-STIM and r-STIM stimulus temporal profiles, and with or without an additional kernel accounting for left-right input interactions (i.e., b-STIM profile as an independent regressor) (Supplementary Figures 7A and 7B, also see Methods). We found that a GLM with the interaction kernel outperformed that without in terms of goodness-of-fit for the majority of neurons (Supplementary Figure 7C), indicating nonlinear interactions of the afferent input signals. Fitting GLMs with an additional kernel to account for the influence of activity history and calcium signal decay revealed similar results (Supplementary Figures 7D-F).

We further characterize the linearity of bilateral integration by determining the fraction of nonlinear information (i.e., fraction of information gained beyond linear summation, denoted by $\left.F_{I s}\right)$ in larvae with unbiased responses to unilateral stimulation $(n=$ 4). $F_{\text {IS }}$ was defined as $\left(I_{\mathrm{S} \_ \text {b-STIM }}-I_{\text {S_u-STIM_sum }}\right) / I_{\text {S_b-STIM }}$, where $I_{\text {S_b-STIM }}$ denotes the mutual information of b-STIM-evoked responses and b-STIM stimulus profile, while $I_{\text {S_u-STIM_sum }_{-}}$ denotes that of linearly summed unilaterally-evoked responses (see Methods). In principle, a neuron can have sublinear $\left(F_{I \mathrm{~s}}<0\right)$, near-linear $\left(F_{I \mathrm{~s}} \approx 0\right)$ or supralinear $\left(F_{I \mathrm{~s}}>0\right)$ gain of information with input summation. Interestingly, apart from observing a supralinear gain of information for the majority of neurons (Figures 6D-G), there is an increasing trend of $F_{\text {Is }}$ (Figures 6F and 6G) as $I_{\mathrm{S}}$ values decrease across brain regions along the rostral-caudal axis (Figures 5A-D). Surprisingly, bilateral cadaverine stimulation appeared to also enhance the $I_{\mathrm{S}}$ values of OSNs in the OE, although to a much lesser extent than in the downstream regions (Figures 6E-G). Additional analysis on right eye-ablated larvae with biased responses to r-STIM $(n=5)$ revealed similar results on information gain in the OE and a similar $F_{I \mathrm{~s}}$ trend across brain regions (Supplementary Figures 8A-C). 
We speculated that the $F_{I \mathrm{~s}}$ increase in the higher-order olfactory processing centers and the caudal brain regions may be due to a larger relative information gain in neurons that weakly encode unilateral olfactory input, which could be more reliant on the convergence of the bilateral inputs. Consistent with the hypothesis, we found a strong inverse correlation between $F_{I \mathrm{~S}}$ and the $I_{\mathrm{S}}$ with preferred OP stimulation $\left(I_{\mathrm{S}_{-} \text {Pref }}\right.$, defined as $\max \left(I_{\mathrm{S}_{-} \text {-STIM }}, I_{\mathrm{S}_{-} \text {-STIM }}\right)$, where $I_{\mathrm{S}_{-} \text {I-STIM }}$ and $I_{\mathrm{S}_{-} \text {-STIM }}$ denote $I_{\mathrm{S}}$ of 1-STIM and r-STIM conditions, respectively) (Figure 6H). Interestingly, in the higher and caudal brain regions, the relationship between $F_{I \mathrm{~s}}$ and $I_{\mathrm{S}_{\text {Pref }}}$ becomes even stronger, whereby the magnitude of the negative slope of linear regression progressively increases (Figures 6I and 6J). This indicates that in these brain regions, given the same unit change in $I_{\mathrm{S}_{-} \text {Pref }}$ there is a relatively larger change of $I_{\mathrm{S}_{-} \mathrm{b}-\mathrm{STIM}}$ (Figures 6I and 6J). We noted the same phenomena in the larvae with biased responses to r-STIM (Supplementary Figures 8D-F). Synergistic input summation may therefore be especially important for sensory information representation by neurons otherwise only weakly encode unilateral input in the downstream brain regions of the olfactory processing pathways. These include the caudal brain regions where the motor-encoding neurons are located (Figures 5E-H).

\section{Bilateral input enhances sensorimotor unit responses in motor-encoding brain regions}

Sensorimotor transformation requires sequential activation of neurons embedded in neural circuits specializing in roles from detection of sensory cues to driving motor outputs. The enhanced sensory representation by bilateral olfactory inputs in brainwide regions should in principle be utilised by subsets of neurons mediating sensorimotor transformation and motor output. We thus examined how sensorimotor neurons may be modulated by unilateral vs. bilateral OP cadaverine stimulation in the subset of stably imaged larval subjects that were behaviorally responsive $(n=6)$. Sensorimotor neurons were defined as those that at least moderately encode both cadaverine stimulus information and motor output (see Methods). Rh had the largest number of sensorimotor neurons, followed by Di and then $\mathrm{Me}$ (Figures 7A and 7B). Most of these units exhibited supralinear sensory information gain with bilateral olfactory inputs, with $F_{I \mathrm{~s}}$ higher than their sensory-encoding-only counterparts in the same regions (Figure 7C). This was also substantiated by examining their regionally averaged responses, as b-STIM resulted in larger stimulus-locked responses than unilateral stimulation (Figures 7D and 7E). We thus concluded that in the sensorimotor transformation process, the bilateral olfactory input-enhanced sensory representation is associated with a stronger drive to the downstream sensorimotor and subsets of motor-encoding neurons (Figure 8).

\section{Discussion}

Considerable progress has been made in circuit neuroscience in the past decade, in part owing to methodological advancements permitting large-scale cellular-resolution imaging of neuronal activities in diverse brain areas in various species, including zebrafish (Ahrens et al., 2012, 2013; Panier et al., 2013; Prevedel et al., 2014), Drosophila (Aimon et al., 2019; Lemon et al., 2015), and rodents (Sofroniew et al., 2016; Stirman et al., 2016; Tsai 
et al., 2015). Whole-brain imaging in larval zebrafish with several microscopic techniques, whereby the activities of as many as nearly $80 \%$ of all neurons could be imaged (Ahrens et al., 2012, 2013; Panier et al., 2013; Prevedel et al., 2014), opened up the avenue for the study of brainwide circuit dynamics (Loring et al., 2020; Mu et al., 2020; Vanwalleghem et al., 2018). It also permitted the assumption-free identification of brain regions mediating different behaviors. Taking advantage of the approach, in this work we developed microfluidics-based methodologies to empower the investigation of chemosensory-mediated behaviors and its neural basis in the larval zebrafish. These included a navigation behavioral assay with a precisely defined and static chemical landscape, and an odorant delivery methodology with high spatiotemporal precisions for the interrogation of whole-brain neural representations. These tools were indispensable to reveal bilateral olfactory input-dependent and independent avoidance behavioral changes to cadaverine, as well as the neural basis of parallel sensory input integration and subsequent sensorimotor transformation in the vertebrate model.

Cadaverine is a chemical of the diamine class of compounds and an aquatic odor of ecological significance. In several species, diamines are detected by a family of trace amine-associated receptors (TAARs) (Liberles, 2015). The zebrafish genome has 109 TAAR genes (Hashiguchi and Nishida, 2007). The adult zebrafish have been shown to express TAAR13c (a TAAR) in a subset of ciliated OSNs that mediate their cadaverine avoidance behavior (Dieris et al., 2017; Hussain et al., 2013). Intriguingly, in a previous study, puff-application of cadaverine did not seem to elicit immediate escape response in zebrafish larvae (Koide et al., 2018). By allowing larvae to navigate in a nature-mimicking arena with precisely defined and continuous chemical-carrying laminar flows, our assay captured the behavioral responses and subsequent adaptation upon naturalistic encounters of cadaverine. Repeated sampling of a large number of cadaverine zone entry events and escape journeys enabled us to reveal adaptive behavioral changes in navigation strategy that occur in a statistical manner.

Although still in early developmental stage, the larval zebrafish has a rich behavioral repertoire with complex dynamical properties. Its spontaneous locomotor activity is characterized by discrete swim bouts with correlated turn-direction bias in successive bouts (Dunn et al., 2016; Marques et al., 2018). The adaptive nature of locomotion is exemplified by at least thirteen swimming patterns used in different combinations depending on the behavioral contexts (Marques et al., 2018). For instance, light modulates locomotion, and zebrafish larvae predominantly swim in slow bouts in darkness (Marques et al., 2018). In our experiments, to avoid the potential confounding effects of light and other visual stimuli, and to isolate the effects of odor stimuli, we carried out the navigation behavioral assays in the absence of visible light. Our data supports that under such context, the larval zebrafish employs a combined strategy of klinokinesis and angular orthokinesis, without directional bias, to efficiently evade cadaverine. It appears to be a sensible strategy, given the small spatial separation of the OPs at this stage likely renders chemical gradient determination by inter-OP comparison sensitive to random fluctuations or noises in sampling. It is yet to be 
addressed, whether with further development, zebrafish may learn to extract and utilize inter-OP odor arrival time or intensity differences for more efficient odorant localization like larger mammals (Catania, 2013; Liu et al., 2020; Porter et al., 2006; Rajan et al., 2006).

Interestingly, we found a differential dependence of klinokinesis and angular orthokinesis on the integrity of bilateral olfactory input. This suggests that cadaverine sensing with a single OP does not pose a problem of detection sensitivity. Rather, different aspects of adaptive navigation behavioral changes may be driven by different neural mechanisms that require different sensory activation thresholds. It may serve as a fail-safe design, as with klinokinesis alone, we observed that the larvae still managed to reduce their exposure to cadaverine in the navigation assay. It remains unclear what may account for the bilateral input-dependent adaptation of turn duration (and thereby turn magnitude). Adaptation of sensorimotor transformation, glial and neuromodulatory system-mediated behavioral futility with successive unsuccessful attempts (Andalman et al., 2019; Mu et al., 2019), and downstream motor output adaptations (e.g., at neuromuscular or muscular levels) could in theory all contribute to the phenomenon. Nonetheless, its presence suggests that once a larval subject fails to evade in the initial swim bouts, subsequent attempts may become less efficient. Therefore, it is behaviorally even more pertinent to perform the first few turns with sufficiently large angle and displacement upon entering a noxious or even toxic chemical-carrying stream.

To study the neural basis of bilateral input integration that underlies the adaptive behavioral changes to cadaverine sensing, the prerequisite is a method for spatiotemporally precise delivery of odor stimuli to unilateral or bilateral OPs. This requires partitioning of the odor delivery fluid streams at ten-micrometer precision. Careful direction of fluid flows around a larva subject is therefore needed, which is achievable by microfluidics techniques with suitable fluid channel geometry design and fluidic pressure control. In our setup, we harnessed the laminar property of fluid flow at low Reynolds number, such that distinct pairs of fluid streams are separately directed to the OPs from the sides and converge at the midline with minimal mixing. With a spatial separation of $\sim 100 \mu \mathrm{m}$ between the two OPs, the maximal tolerable deviation of midline without contacting the opposite OPs is approximately $\pm 50 \mu \mathrm{m}$, well above the typical length scale of microfluidic flow $(\sim 5 \mu \mathrm{m})$. In order to minimize the delay of fluid changes which is intrinsically variable (subject to momentary local fluidic resistance), we aimed to switch fluids as close to the larva as possible. We therefore used insulating water streams between the OPs and the cadaverine streams, which can be rapidly switched off for repeated trials of temporally reproducible odor delivery.

With obvious ecological significance, the olfactory system and its associated pathways develop early in the zebrafish. Within 7 d.p.f., the OB projection pathways that target diverse forebrain regions are already formed, which include extensive inter-hemispheric projections (Kunst et al., 2019; Miyasaka et al., 2014). Although we observed a small overall bias of neuronal responses to ipsilateral OP cadaverine stimulation, many neurons in each of the brain regions are equally driven by the left and the right stimuli. 
This suggests that while the ipsilateral project pathways may be slightly stronger, neither the ipsilateral nor the contralateral projection pathways functionally dominate. The functional roles of commissural pathways likely vary depending on the specific computational goals to achieve (Gebhardt et al., 2019; Kermen et al., 2020; Yan et al., 2008). In the adult zebrafish, the interbulbar pathways serve to improve the detection of a reproductive pheromone especially when there are co-existing confounding odor cues (Kermen et al., 2020). In cadaverine sensing, we found that bilateral olfactory input convergence confers a generally supralinear sensory information gain in the OBs and downstream regions (Figure 8). The interbulbar and the other commissural olfactory pathways are therefore already functional at the larval stage, and mediate overall excitatory or facilitatory interactions, as we observed predominantly positive response modulation by the odor stimuli. Our results also suggest that they likely serve to improve the signal-to-noise ratio of odor detection, and enhance subsequent sensorimotor transformation in cadaverine avoidance (Figure 8). Note however, despite the early signal convergence, a wide range of response selectivity to each of the OP inputs is maintained in the higher-order olfactory processing centers and the more caudal regions (Figure 8). This distributed representation preserves information on sensory cue laterality. If conserved with further development, the design may provide the neural substrate for juvenile or adult zebrafish to potentially utilize the information for guiding directed turns, thereby further optimizing navigation.

Originally intended to improve the optical accessibility of forebrain regions, we unexpectedly observed biased responses to the ipsilateral OP in the majority of larvae imaged after right eye ablation. The absence of right eye stimulation appeared to potentiate the responses to ipsilateral olfactory stimulus and decrease that to the contralateral side. There are several possible explanations for the crossmodal response modulation. A potential mechanism is lateralized sensory compensation (Hartman and Abrahams, 2000), whereby removal of the right eye may have led to a heightened odor sensitivity of the ipsilateral OE, while a yet-to-be characterized inhibitory pathway may suppress that of the contralateral counterpart. To circumvent the phenomenon, future works will need refined microfluidic device design that can simultaneously incorporate fluid inlets and an additional narrow light sheet from the front to illuminate the forebrain, while the light sheet entering from the side can then conveniently avoid the eyes (Vladimirov et al., 2014).

Although the $\mathrm{OE}$ is generally considered to be a purely sensory station without descending modulations, we surprisingly observed weak OSN response enhancement by contralateral olfactory stimulation. In the mouse, a couple of recent works reported brain state-dependent modulation of retinal ganglion cell output in the superior colliculus (Liang et al., 2020; Schröder et al., 2020). Similarly, associative learning influences OSN synaptic release in the mouse olfactory bulb (Bhattarai et al., 2020). Candidate mechanisms proposed include top-down or local circuit-mediated presynaptic modulation at the axonal terminals (Bhattarai et al., 2020), or retinopedal projections-mediated top-down influence directly at the retina (Liang et al., 2020; Schröder et al., 2020). These hypotheses have the necessary anatomical substrates to support in the mouse. However, in the larval zebrafish, current 
anatomical data has not shown any direct inter-hemispheric or top-down projections to the OE (Kunst et al., 2019; Miyasaka et al., 2014). Alternatively, this may be achieved via glia-mediated neuronal response modulation. If further consolidated, we propose that such modulation of primary OSN responses by contralateral input could also aid in enhancing the detection and amplification of odor signals, which is especially important for sensing danger-signaling cues.

Finally, the microfluidics-based behavioral, chemical delivery and imaging platforms we developed can be readily adapted for studying chemosensory behaviors in other organisms of similar or smaller length scales, such as bacteria, C. elegans (see related works by (Chronis et al., 2007; Levy and Bargmann, 2020)), Drosophila larvae and adults. In fact, other fluid properties (e.g., temperature, viscosity) can also be precisely controlled. Convenient incorporation of crossmodal stimuli allows the study of their combined effects on the behaviors and neural responses of animal subjects. On the other hand, the high-precision delivery system is extensible to accommodate an elaborate array of chemicals varying in species, concentrations and valence, to interrogate the corresponding neural representations in the larval zebrafish. In this work, by delivering congruent odors to the OPs, we showed how bilateral input integration may permit odor perceptual enhancement. On the other hand, by delivering incongruent odors with opposite valence to the $\mathrm{OP}(\mathrm{s})$, one may investigate how competing circuits implement the prioritization of ecologically more important sensory cues. These will gain us deeper insights into the architecture of odor information processing in vertebrates. 


\section{Acknowledgements}

We thank Tom Mrsic-Flogel and Florian Engert for helpful comments on the manuscript; Jan Schnupp and Vincent Cheung for insightful discussions; Florian Engert for provision of the Tg(elav13:GCaMP6f) zebrafish. We thank Becky Yung, Florence Yau, Anki Miu and Rebecca Chau for administrative support to the project. This work was in part funded by a Croucher Innovation Award from the Croucher Foundation and a Faculty Innovation Award (FIA2017/B/01) from the Faculty of Medicine, the Chinese University of Hong Kong (CUHK) (H.K.); the Gerald Choa Neuroscience Centre, the Margaret K. L. Cheung Research Centre for Parkinsonism Management, Faculty of Medicine, CUHK (V.C.T.M. and H.K.); the Collaborative Research Fund (C6027-19GF) and the Area of Excellence Scheme (AoE/M-604/16) of the University Grants Committee of Hong Kong (H.K.).

\section{Author Contributions}

S.K.H.S. constructed experimental setups, carried out experiments and analyzed data. D.C.W.C. contributed to image processing. H.M.L. contributed to experimental protocol testing. Z.L. assisted two-photon ablation experiments. K.K.Y.W., C.H.J.C. and V.C.T.M. contributed to data interpretation. Y.H. contributed to data analysis. S.K.H.S., D.C.W.C. and H.K. wrote the manuscript with input from all authors.

\section{Declaration of Interests}

The authors declare no competing interests. 


\section{STAR $\star$ METHODS}

\section{KEY RESOURCES TABLE}

\begin{tabular}{|c|c|c|}
\hline REAGENT or RESOURCE & SOURCE & IDENTIFIER \\
\hline \multicolumn{3}{|l|}{ Chemicals } \\
\hline Cadaverine & Sigma & D22606-5G \\
\hline \multicolumn{3}{|c|}{ Experimental Models: Organisms/Strains } \\
\hline $\begin{array}{l}\text { Tg(elav13:GCaMP6f) } \\
\text { zebrafish larvae }\end{array}$ & $\begin{array}{l}\text { Engert lab } \\
\text { (Harvard) }\end{array}$ & N/A \\
\hline \multicolumn{3}{|l|}{ Software and Algorithms } \\
\hline $\begin{array}{l}\text { Custom codes written in } \\
\text { MATLAB R } 2018 b\end{array}$ & This paper & $\underline{\text { https://www.mathworks.com/products }}$ \\
\hline AutoCAD 2018 & Autodesk & $\begin{array}{l}\underline{\text { https://www.autodesk.com/products/a }} \\
\underline{\text { utocad/overview? support=ADVANC }} \\
\underline{\text { ED }}\end{array}$ \\
\hline StreamPix & Norpix & $\begin{array}{l}\text { https://www.norpix.com/products/stre } \\
\underline{\text { ampix/streampix.php }}\end{array}$ \\
\hline CFDTool & Precise Simulation & $\begin{array}{l}\underline{\text { https://github.com/precise-simulation/ }} \\
\underline{\text { cfdtool/raw/master/CFDTool.mlappin }} \\
\underline{\text { stall }}\end{array}$ \\
\hline $\begin{array}{l}\text { Variational Stationary } \\
\text { Noise Remover }\end{array}$ & $\begin{array}{l}\text { (Fehrenbach et al., } \\
\text { 2012) }\end{array}$ & $\begin{array}{l}\underline{\text { https://github.com/sattarab/image-qua }} \\
\underline{\text { lity-tools/tree/master/metrix_mux/met }} \\
\underline{\text { rix/vsnr }}\end{array}$ \\
\hline NoRMCorrE & $\begin{array}{l}\text { (Pnevmatikakis and } \\
\text { Giovannucci, 2017) }\end{array}$ & $\begin{array}{l}\text { https://github.com/flatironinstitute/No } \\
\underline{\text { RMCorre }}\end{array}$ \\
\hline CaImAn & $\begin{array}{l}\text { (Giovannucci et al., } \\
\text { 2019) }\end{array}$ & $\begin{array}{l}\underline{\text { https://github.com/flatironinstitute/Ca }} \\
\underline{\text { ImAn-MATLAB }}\end{array}$ \\
\hline Fiji - ImageJ & $\begin{array}{l}\text { (Schindelin et al., } \\
\text { 2012) }\end{array}$ & $\underline{\text { http://fiji.sc }}$ \\
\hline
\end{tabular}




\section{RESOURCE AVAILABILITY}

\section{Lead Contact}

Further information and requests for resources and reagents should be directed to the lead contact, Ho Ko (ho.ko@cuhk.edu.hk).

\section{Materials Availability}

The AutoCAD files of the microfluidic devices developed in this study are available upon reasonable request to the lead contact.

\section{Data and Code Availability}

Preprocessed data necessary to replicate these results will be deposited to G-Node, and the MATLAB code for data analysis will be made available on GitHub upon the acceptance of the paper.

\section{EXPERIMENTAL MODEL AND SUBJECT DETAILS}

All experimental procedures were approved in advance by the Animal Research Ethical Committee of the Chinese University of Hong Kong (CUHK) and were carried out in accordance with the Guide for the Care and Use of Laboratory Animals. For all experiments reported in this study, we used 5-7 days post fertilization (d.p.f.) larvae carrying the nacre mutation. Larvae were raised and maintained under 14-h light:10-h dark cycles at $28^{\circ} \mathrm{C}$. The larvae used for the bilateral OP-intact control and cadaverine groups in the navigation behavioral assay were bred from a pair of heterozygous Tg(elav13:GCaMP6f) adult zebrafish without screening for GCaMP6f expression. For the unilateral OP-ablated groups, the larvae used in the assays had confirmed GCaMP6 $f$ expression (by examination under an epifluorescence microscope) to allow structural visualization and two-photon OP ablation.

\section{METHOD DETAILS}

\section{Microfluidic arena for chemosensory behavioral assays}

The microfluidic device for the avoidance navigation assay was designed in AutoCAD 2018 (Autodesk, USA) and consists of three $1.5 \mathrm{~mm}$-thick laser-engraved poly(methyl methacrylate) (PMMA) layers. The layers were vertically aligned and fused by a chloroform solution applied to the edges of the sheets. This created a closed rectangular arena $(60 \mathrm{~mm}(\mathrm{~L}) \times 30 \mathrm{~mm}(\mathrm{~W}) \times 1.5 \mathrm{~mm}(\mathrm{H}))$ connected to three fluid inlets and two fluid outlets during assays (Figure 1A). An additional inlet was used for loading larvae and sealed prior to assays. The inlets and outlets are $200 \mu \mathrm{m}$ wide, prohibiting larvae from exiting the arena through these channels. Syringe needles (Terumo, USA) were sealed at the top layers with epoxy adhesive paste (Devcon, USA) to connect the inlet and outlet channels to fluid-filled syringes and waste collection bottle, respectively, via poly(tetrafluoroethylene) (PTFE) tubes (Cole Parmer, USA). The microfluidic device was then air-dried for one day to allow 
evaporation of the residual chloroform and epoxy adhesive paste. The device was rinsed twice with water before use for behavioral assays.

To create a static chemical zone, three fluid streams (two water streams and one odor stream with $1 \mathrm{mM}$ cadaverine dissolved in water) were formed in the arena by propelling the respective fluids through the inlet channels at $220 \mu \mathrm{L} / \mathrm{min}$ using syringe pumps (LSP02-2A, Longerpump, Beijing) (Figure 1A). The outlets were connected to open waste collection bottles. The Reynolds number $(R e)$ of fluid flow in the arena is given by $R e=\rho v D_{\mathrm{H}} / \mu$, where $\rho$ is the fluid density, $v$ is the fluid flow velocity, $D_{\mathrm{H}}$ is the hydraulic diameter of the device, and $\mu$ is the fluid dynamic viscosity. Given the rectangular cross-sectional dimensions of the arena (i.e., $1.5 \mathrm{~mm} \times 60 \mathrm{~mm}$ ), the average volumetric flow rate was $220 \mu \mathrm{L} \mathrm{min}^{-1} \times 3$ inlets $=$ $660 \mu \mathrm{L} \mathrm{min}$, which equals $11 \mathrm{~mm}^{3} \mathrm{~s}^{-1}$. Hence the average flow velocity $=11 \mathrm{~mm}^{3} \mathrm{~s}^{-1} /(1.5$ $\mathrm{mm} \times 60 \mathrm{~mm})=1.22 \times 10^{-4} \mathrm{~m} \mathrm{~s}^{-1} . D_{\mathrm{H}}$ for the rectangular channel is given by $2 a b /(a+b)=9$ $\times 10^{-5} \mathrm{~m}$, where $a$ and $b$ are the dimensions of the rectangular cross section (i.e., $1.5 \mathrm{~mm}$ and $60 \mathrm{~mm})$. Substituting the $D_{\mathrm{H}}$ found and the density $\left(996 \mathrm{~kg} \mathrm{~m}^{-3}\right)$ and dynamic viscosity $(8.32$ $\times 10^{-4} \mathrm{~m}^{2} \mathrm{~s}^{-1}$ ) of water at $28^{\circ} \mathrm{C}$, the Reynolds number was found to be $R e=0.013$. As $R e<<$ 2000 , the streams were laminar with negligible mixing (Rhodes, 1989) and static fluid zone borders were formed (Figure 1A, Supplementary Figure 1B). The static-border property of the chemical zone during the entire 2-hour assay period was validated by infusing an infrared dye (IR 806, Sigma, USA) into the rightmost stream of the device (Supplementary Figure 1B).

During experiments, the device was levelled and the outflow rates of the two outlets were ensured to be identical. The two-dimensional (2D) flow velocity profile (Supplementary Figure 1A) was obtained by computational fluid dynamics simulation using the CFDTool toolbox (Precision Simulation) in MATLAB (R2018b, MathWorks, USA), assuming incompressible Newtonian fluid flow and with a grid size of $1.4 \times 10^{-4} \mathrm{~m}$. The geometry and boundary conditions were set according to the aforementioned device parameters.

\section{Chemosensory avoidance navigation and survival assays}

During a chemosensory avoidance navigation assay, three to six larvae aged 5-7 days d.p.f. were placed in a swimming arena and allowed to acclimate for 15 minutes. Swimming and navigational behaviors were then recorded by a high-speed camera at $240 \mathrm{fps}$ (Mako G-030B PoE, Allied Vision, Germany) under 850-nm IR illumination (ANGX-1000-CH1-24V, TMS, Malaysia) for 2 hours in the absence of visible light. Fluid temperature was maintained at $\sim 28^{\circ} \mathrm{C}$ throughout the assay.

For the control group, all three fluid streams consisted of water. For the cadaverine zone avoidance assay groups, the rightmost stream contained $1 \mathrm{mM}$ cadaverine (Sigma, USA) dissolved in water (Figure 1A), which was freshly prepared prior to each experiment due to the evaporative nature of cadaverine. For the left or right OP-ablated groups, larvae aged 4-5 d.p.f. were first individually immobilized in 1.5\% low-melting agarose (Sigma, 
USA), then subjected to two-photon ablation of the OP which was performed under a two-photon microscope (Scientifica, UK) with a Ti:sapphire femtosecond laser (Spectra-Physics, USA) tuned to $830 \mathrm{~nm}$. The unilateral OP-ablated larvae were then allowed to recover for at least 24 hours prior to experiments. Before the behavioral assays, the OPs were imaged again under the two-photon microscope to ensure successful ablation (see the left panels of Figures 1C and 1D). The numbers of assays, larvae and border-crossing event sample sizes for the four experimental groups are as follows: bilateral OP-intact larvae (control group without cadaverine zone, i.e., all water zones): 6, 24, 120; bilateral OP-intact larvae (assay group with cadaverine zone): 8, 32, 128; left OP-intact larvae (assay group with cadaverine zone): 4, 15, 37; right OP-intact larvae (assay group with cadaverine zone): 4, 15, 98.

For the survival assays, larvae were individually put in a device filled with $1 \mathrm{mM}$ cadaverine dissolved in water without flow. Videos of the larvae were recorded at $10 \mathrm{fps}$, and stimulated with gentle taps to the device every 15 minutes. The assay was terminated when the larva became unresponsive and death was confirmed by the absence of heartbeats and circulation under microscopic examination. No larvae survived continuous cadaverine exposure more than 165 minutes. A control group of larvae underwent the same assay with devices filled with water for 180 minutes.

\section{Microfluidic device for precise olfactory placode stimulation, behavioral and brainwide imaging}

Studying bilateral olfactory input integration in larval zebrafish requires fine spatiotemporal control of chemical-carrying fluid flow around the larval subject. On the other hand, stable cellular-resolution neuronal imaging of partially restrained larval zebrafish wholly immersed in continuous fluid medium during OP-specific stimulus presentation must be attained. The PDMS-based microfluidic devices developed to achieve these goals (Figure 4A and Supplementary Figure 3A) were designed in AutoCAD 2018 (Autodesk, USA). Slight customizations were made to the larva head and waist-trapping chambers to allow the fitting of either a right eye-ablated or right-tilted larva. The microfluidic device was fabricated by customized photolithography techniques to incorporate a flat and smooth sidewall for excitation light sheet entry, and a thin glass ceiling for emitted fluorescence detection path (Supplementary Figure 3A). Briefly, a 2D design was printed on a soda lime mask (Supermask, Shenzhen). Negative photoresist SU-8 2150 (Microchem, USA) was spin-coated on a 4 -inch silicon wafer (Semiconductor Wafer, Taiwan) to $500 \mu \mathrm{m}$-thick. The device pattern was then transferred to the photoresist with UV exposure (OAI, USA), followed by post-exposure bake and etching to produce a master mold. Mixed PDMS (Dow Corning, USA) and curing agent (at 10:1 w/w) was poured into the salinized mold to a thickness of $5 \mathrm{~mm}$, with a perpendicular glass slide held upright on the mold to produce a smooth vertical surface upon curing (Supplementary Figure 3A). After solidification at $100^{\circ} \mathrm{C}$ for 60 minutes, it was detached from the mold, bonded and sealed onto a microscopic 
cover slide (as thin glass ceiling) by plasma treatment. The devices were rinsed with system water twice before use in experiments.

We derived a fluidic stream control solution to achieve the necessary spatial (Supplementary Figure 3B) and temporal precisions of olfactory stimulation (Supplementary Figure 3C). On each side anterior to the larva, there were sandwiched laminar fluid streams (water-cadaverine-water) which converged at the midline. The cadaverine stream consisted of $1 \mathrm{mM}$ cadaverine dissolved in water, with $1 \mu \mathrm{M}$ sodium fluorescein for the visualization of chemical delivery. Each fluid stream was carried by PTFE tubes, controlled by an solenoid valve (LHDA 0533115H, Lee Company, USA) and driven by a syringe pump. The posterior water streams on each side insulates the OPs from contacting the corresponding cadaverine streams (Figure 4C, Supplementary Figure 3C, left). Closure of the valve of the insulating water stream on one side removes the water barrier to the chemical and enables precise chemical stimulation of the ipsilateral OP (Figure 4C, Supplementary Figure 3C, middle). Unintended stimulation of the contralateral OP is prevented by the presence of opposing flow on the contralateral side (Figure 4C, Supplementary Figure 3C, middle). Stimulation is turned off by closure of the chemical stream valves (Supplementary Figure 3C, right). Bilateral stimulation is achieved by closure of the insulating water stream valves on both sides (Figure 4C) and terminated by closure of the chemical stream valves. A side channel connecting the chemical delivery microchannels and the tail chamber was incorporated to buffer pressure changes in the fluidic environment, and minimize mechanical disturbances to the larval subject. Flow rate of each stream were maintained at $88 \mu \mathrm{L} / \mathrm{min}$ with slight adjustments for each larva to compensate for the differences in position in the device, ensuring precise convergence of the opposing fluid streams at the midpoint between the OPs. Absence of cadaverine spillover to the contralateral side was confirmed for each experimental trial by $>20: 1$ (ipsilateral:contralateral) fluorescence intensity changes in the pixels immediately in front of the ipsilateral and contralateral OPs (also see the subsequent section on image processing and calcium signal extraction on the extraction of 1-STIM and r-STIM stimulus profiles).

\section{Light sheet microscope for whole-brain calcium imaging}

We used a custom-built light sheet microscope for cellular-resolution whole-brain imaging (Figure 4). A 486 nm-centered blue gaussian laser (DL-488-050-0, Crystalaser, USA) was used as the excitation light source. The laser beam was resized to $0.6 \mathrm{~mm}$ in diameter $\left(1 / e^{2}\right)$ by a pair of telescopic lenses (LB1757-A, LB1596-A, Thorlabs, USA), which then passed through a scanning system, followed by a cylindrical lens (LJ1695RM-A, Thorlabs, USA) that focused the horizontal dimension of the parallel beam onto the back focal plane of an air excitation objective (Nikon Plan Fluorite, $\times 10$, N.A. 0.3, $16 \mathrm{~mm}$ WD). This expanded the laser horizontally to form a light sheet. The scanning system consisting of a galvanometric mirror (GVS211/M, Thorlabs, USA), a F-theta lens (S4LFT0061/065, Sill Optics, Germany) and a tube lens (TTL200-B, Thorlabs, USA) was used to scan the light sheet vertically and linearly over a range of $210 \mu \mathrm{m}$. The F-theta and tube lens also expanded 
the beam 3.31 times, resulting in a beam diameter of $2 \mathrm{~mm}\left(1 / 9^{\text {th }}\right.$ of the objective back aperture) and effective excitation N.A. of 0.0332 . Thus, a thin excitation sheet (thickness: $7.33 \mu \mathrm{m}\left(1 / e^{2}\right)$, Rayleigh length: $\left.\sim 300 \mu \mathrm{m}\right)$ was generated.

Along the detection path, a bandpass green filter $(525 \pm 25 \mathrm{~nm})$ was placed after an air detection objective (Nikon Plan Fluorite, $\times 10$, N.A. 0.3, $16 \mathrm{~mm}$ WD) to block the blue excitation light. An electrically tunable lens (EL-10-30-Ci-Vis-LD, Optotune, Switzerland) in between a pair of relay lenses (LA1509-A, Thorlabs, USA) was linearly driven by a lens controller (TR-CL-180, Gardasoft, UK) and synchronized with the light sheet scanner to achieve rapid focusing of different image planes onto the sensor of a sCMOS camera (Zyla 5.5, Andor, UK). During an experiment, to correct for axial drift, a reference plane was calibrated with respect to the initial measurement at the beginning of each trial. All control units were synchronized using a multifunctional I/O device (PCIe-6323, National Instruments, USA).

\section{Simultaneous whole-brain calcium imaging and tail flicking behavior recording}

A total of eighteen 5-6 d.p.f. right eye-ablated and four tilted zebrafish larvae underwent simultaneous behavioral and whole-brain calcium imaging experiments. For the right eye-ablated group, 4.5-5 d.p.f. larvae were lightly anesthetized in $0.016 \%$ MS-222 and the right eyes were gently removed using surgical forceps under a dissection microscope. Larvae were returned to warmed Ringer's solution and allowed to recover for 12-24 hours before imaging experiments.

In an imaging experiment, a single larva was loaded and fitted into the trapping chamber of the PDMS-based microfluidic device using a syringe pump and monitored under a surgical microscope. The larvae inlet was then sealed and the larva was allowed to acclimate for 15 minutes. The axial focus of the light sheet was adjusted to be centered at the brain, correcting for small variations in side wall width and larva position inside the device. Prior to functional imaging, a detailed anatomical stack of the larval zebrafish brain spanning 138 imaging planes at 2- $\mu \mathrm{m}$ intervals was taken.

Whole-brain calcium imaging spanning 29 planes at 7- $\mu \mathrm{m}$ intervals was performed at $2 \mathrm{~Hz}$ volumetric rate, with each trial lasting for 50 or 70 seconds, and $\sim 90$-second inter-trial intervals. Trials consisted of 30 -second baseline, 10 - or 25 -second stimulus presentation, and 10 - or 15-second post-stimulus epochs. In between the trials, a resting interval was followed by axial drift correction. Each larva underwent 3-4 trials of null stimulus condition recordings and 3-6 trials of each cadaverine stimulus condition (i.e., left, right or bilateral OP stimulation). During each trial, after a 30 -second baseline recording period, the first (water) and the second (cadaverine) stream in front of the OP(s) were switched off sequentially with 10 - or 25-second gap to allow the second (cadaverine) followed by the third stream (water) to contact the $\mathrm{OP}(\mathrm{s})$, resulting in the initiation and termination of olfactory stimulation, respectively (Supplementary Figure 3C). Recording continued for 10 or 15 seconds after 
stimulus cessation. Simultaneous tail flicking behavior was imaged at $200 \mathrm{fps}$ under IR illumination by a CCD camera (F032B, Pike, Germany).

\section{QUANTIFICATION AND STATISTICAL ANALYSIS}

\section{Navigational behavior analysis}

Individual frames of the navigation behavioral tracking videos were first registered for translation, background-subtracted, and contrast-adjusted. The heading orientation $\left(10^{\circ}\right.$ resolution) and $x y$-coordinates $(0.1 \mathrm{~mm}$ resolution) of every larva in each frame were extracted using a semi-automated template matching-based tracking program custom-written in MATLAB. All tracking results were manually verified on a frame-by-frame basis and corrected when necessary. When larvae came in close proximity $(\leq 4 \mathrm{~mm})$ to either the arena boundary or with each other, the trajectories were excluded from analysis of turn behaviors to rule out potential social or mechanical cue-associated movements. A cadaverine-water border was defined with the aid of an IR dye (IR 806, Sigma, USA) in a separate assay (Supplementary Figure 1B). A 1-mm margin along the border was incorporated in analysis, to account for the minimal diffusion and mixing. Cadaverine zone border-crossing events were identified when swim bout trajectories intersected the cadaverine-water border from the water zone. Subsequent escape trajectories were isolated and analysed to compare swim bout kinematic parameters and escape efficiency for the different experimental groups.

\section{Analysis of tail flicking behavior}

Each frame of tail flicking behavioral imaging was background-subtracted and the tail tip-waist angle was extracted by a custom MATLAB program. The tail tip-waist angle was defined as the angle between the long axis of the larval subject body and a straight line joining the tail tip and the waist. The extracted angles were manually verified on a frame-by-frame basis and used for the calculations of tail flick event frequency and angular velocity.

\section{Image processing and calcium signal extraction}

Detailed larval zebrafish brain anatomical stacks were registered to the Z-Brain atlas (Randlett et al., 2015) using affine transformation followed by non-rigid image registration using custom scripts written in MATLAB. Functional imaging planes were matched to the corresponding anatomical stack by maximization of pixel intensity cross-correlation and manually verified. Stripe artefacts in the anatomical stacks and functional imaging frames were removed using the Variational Stationary Noise Remover (VSNR) algorithm (Fehrenbach et al., 2012). Functional imaging frames were motion-corrected using the NoRMCorrE algorithm (Pnevmatikakis and Giovannucci, 2017). Trials with blurred frames due to in-frame drifts were discarded. Regions of interest (ROIs) corresponding to individual neurons were then extracted using the CaImAn package (Giovannucci et al., 2019). Anatomical landmarks and the regional identity of each ROI were verified by manual inspection. The stimulus temporal profiles of 1-STIM or r-STIM were obtained as the average 
pixel intensity of a manually defined ROI located immediately anterior to each of the OPs. The stimulus profiles were characterized by a rapid rise $(0.93 \pm 1.13 \mathrm{~s}$ (mean \pm S.D.) to rise from $10 \%$ to $90 \%$ of maximum) reaching a plateau, and a fast decay after the specified stimulus durations $(1.64 \pm 1.04 \mathrm{~s}$ (mean \pm S.D.) to fall from $90 \%$ to $10 \%$ of maximum).

\section{Identification of sensory-encoding, motor-encoding, and sensorimotor neurons}

The first 10.5 second (21 imaging frames) of the imaging data of each trial were excluded from analysis to avoid the inclusion of transient activity evoked by the onset of light sheet illumination. The minimum fluorescence intensity in the subsequent 4.5 seconds ( 9 imaging frames) of each ROI was used as the baseline fluorescence $(F)$. The $d F / F$ signals of remaining imaging frames were calculated as the calcium responses of each ROI. The left $\left(C_{\mathrm{L}, t}\right)$ and right $\left(C_{\mathrm{R}, t}\right)$ cadaverine stimulus profiles were extracted using the abovementioned ROIs directly in front of the respective OPs and normalized to the $[0,1]$ range. The cadaverine stimulus profiles used in bilateral OP-stimulated trials $\left(C_{\mathrm{L}+\mathrm{R}, t}\right)$ were obtained by averaging the left and right stimulus profiles and normalized to the $[0,1]$ range. We calculated the mutual information $\left(I_{\mathrm{S}}\right)$ between the calcium responses of each ROI and cadaverine stimulus profiles under each stimulus condition by a method based on kernel density estimation of the probability density functions of variables (Moon et al., 1995). Each ROI thus had three $I_{\mathrm{S}}$ values, namely $I_{\mathrm{S}_{-} \text {I-STIM }}, I_{\mathrm{S}_{-} \text {-STIM }}$, and $I_{\mathrm{S}_{-} \text {b-STIM }}$, corresponding to $I_{\mathrm{S}}$ values between evoked responses and $C_{\mathrm{L}, t}, C_{\mathrm{R}, t}$ and $C_{\mathrm{L}+\mathrm{R}, t}$, respectively. To estimate the expected distributions of $I_{\mathrm{S} \_ \text {-STIM }}, I_{\mathrm{S} \text { r-STIM }}$, and $I_{\mathrm{S} \text { b-STIM }}$ due to randomness (shuffled $I_{\mathrm{S}}$ ), we randomly shuffled the $d F / F$ signals of each ROI (i.e., disrupting their temporal structures) and calculated another set of shuffled values of $I_{\text {S_l-STIM }}, I_{\text {S_r-STIM }}$, and $I_{\text {S_b-STIM }}$ (with each ROI contributing one value to each of the distributions). We defined sensory-encoding neurons (Figures 5 and 6, Supplementary Figures 6, 7 and 8) to be those that most significantly encode cadaverine stimulus, with at least one $I_{\mathrm{S}}$ value $>2.5$ times the maximum value of the

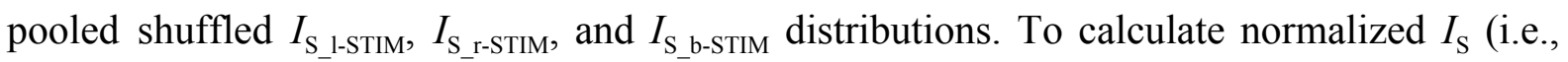
$I_{\mathrm{N}}$ ), the $I_{\mathrm{S}}$ of individual ROIs of a given larva were normalized to the mean $I_{\mathrm{S}}$ of its left, right or bilateral OBs, during 1-STIM, r-STIM or b-STIM, respectively.

For each sensory-encoding ROI, we calculated its right input selectivity, defined as $I_{\mathrm{S} \_ \text {r-STIM }} /\left(I_{\mathrm{S} \text { _-STIM }}+I_{\mathrm{S} \text { r-STIM }}\right)$, where $I_{\mathrm{S} \text { _-STIM }}$ and $I_{\mathrm{S} \text { r-STIM }}$ denote mutual information between calcium responses and left or right $\mathrm{OP}$ stimulus profiles, respectively. We classified the larval subjects to have biased responses if the mean right input selectivity of all sensory-encoding ROIs (except the OE) deviated from a balanced-response value (i.e., 0.5) by more than 0.15 (i.e., $>0.65$ or $<0.35$ ). Those with mean right input selectivity $\geq 0.35$ and $\leq 0.65$ were considered generally unbiased.

We defined motor output to be the proportion of frames (200 fps) with active tail flicking in each 0.5 -s time bins. The calcium response-motor output mutual information $\left(I_{\mathrm{M}}\right)$ was calculated with the same kernel density estimator-based method using data from all stimulus conditions. Shuffled $I_{\mathrm{M}}$ values were calculated using temporally shuffled motor output traces (with each ROI contributing one value to the distribution). Motor-encoding 
neurons (Figure 5) were defined as those that most significantly encode motor output, with $I_{\mathrm{M}}>2.5$ times the maximum values of the shuffled distribution.

Sensorimotor neurons (Figure 7) were defined as those with at least moderate encoding of both sensory (at least one $I_{\mathrm{S}}>1.25$ times maximum of the shuffled $I_{\mathrm{S}}$ values) and motor output $\left(I_{\mathrm{M}}>1.25\right.$ times maximum of the shuffled $I_{\mathrm{M}}$ values) information. Sensory-only neurons (Figure 7) were classified based on strong sensory encoding (at least one $I_{\mathrm{S}}>2.5$ times maximum of the shuffled $I_{\mathrm{S}}$ values) and lack of significant motor encoding $\left(I_{\mathrm{M}} \leq 1.25\right.$ times maximum the shuffled $I_{\mathrm{M}}$ values) (i.e., equivalent to sensory-encoding neurons defined as above with an additional constraint on the lack of motor encoding). Motor-only neurons (Figure 7) were classified based on strong motor encoding $\left(I_{\mathrm{M}}>2.5\right.$ times maximum of the shuffled $I_{\mathrm{M}}$ values) and lack of significant sensory encoding (all $I_{\mathrm{S}} \leq 1.25$ times maximum of the shuffled $I_{\mathrm{S}}$ values) (i.e., equivalent to motor-encoding neurons defined as above but with an additional constraint on the lack of sensory encoding).

For the response traces shown (Figures 6D and 7D, and Supplementary Figures 7B and $7 \mathbf{E}$ ), normalized $d F / F$ traces were calculated by dividing the $d F / F$ values by the maximum of the trial-averaged traces of all stimulus conditions, to facilitate visualization and comparison of each ROI's or region's averaged responses across stimulus conditions.

\section{Analysis of input selectivity and fraction of nonlinear information with bilateral input integration}

Ipsilateral input selectivity for sensory-encoding neurons was defined as $I_{\text {S_ipsi-STIM }} /\left(I_{\text {S_ipsi-STIM }}+I_{\text {S_contra-STIM }}\right)$, where $I_{\text {S_ipsi-STIM }}$ and $I_{\text {S_contra-STIM }}$ denote mutual information between calcium responses and ipsilateral or contralateral OP stimulus profiles, respectively. For a ROI in the left brain, $I_{\mathrm{S} \_ \text {ipsi-STIM }}$ and $I_{\mathrm{S} \_ \text {contra-STIM }}$ were equivalent to $I_{\mathrm{S} \_ \text {-SSTIM }}$ and $I_{\mathrm{S} \_ \text {r-STIM }}$, respectively. Likewise, for a $\mathrm{ROI}$ in the right brain, $I_{\mathrm{S} \_ \text {ipsi-STIM }}$ and $I_{\mathrm{S}_{-} \text {contra-STIM }}$ were equivalent to $I_{\text {S_r-STIM }}$ and $I_{\text {S_l-STIM }}$, respectively.

The fraction of nonlinear information $\left(F_{I S}\right)$ of each ROI was defined as ( $I_{\mathrm{S} \_ \text {b-STIM }}$ $\left.I_{\text {S_u-STIM_sum }}\right) / I_{\text {S_b-STIM }}$, where $I_{\text {S_u-STIM_sum }}$ denotes the mutual information between linearly summed unilateral OP stimulation-evoked responses and $C_{\mathrm{L}+\mathrm{R}, t}$ (see above). The linearly summed unilateral OP stimulation-evoked responses were computed by adding the trial-averaged non-preferred OP (i.e., the OP with lower $I_{\mathrm{S}}$ upon stimulation) stimulation-evoked response trace to each preferred OP stimulation-evoked response traces. For this analysis, either ROIs with $I_{\mathrm{S}_{-} \text {b-STIM }}>2.5$ times the maximum value of shuffled $I_{\mathrm{S}_{-} \mathrm{b}-\mathrm{STIM}}$ (Figure 6 and Supplementary Figure 8) or sensorimotor neurons (Figure 7) were included.

\section{Generalized linear model (GLM) fitting}

For the calcium responses of sensory-encoding neurons, we fitted Lasso regularised and cross-validated generalized linear models (GLMs) with custom scripts written in MATLAB. The regressors in the GLMs included the left $\left(C_{\mathrm{L}, t}\right)$ and right $\left(C_{\mathrm{R}, t}\right)$ cadaverine stimulus profiles, with or without (i) an activity history-dependence regressor, and (ii) a 
bilateral input interaction regressor $\left(C_{\mathrm{L} \cap \mathrm{R}, t}\right.$, defined as the averaged left and right stimulus profile with values outside the time windows of bilateral stimulation set to zero, see Supplementary Figures 7A and 7D). In the simplest GLM (Model I), the calcium response at time $t$ of a given ROI $R_{t}=R_{0}+C_{\mathrm{L}, t} * f_{\mathrm{L}}+C_{\mathrm{R}, t} * f_{\mathrm{R}}$, where $*$ denotes convolution, $R_{0}$ is a $\mathrm{DC}$ term, and $f_{\mathrm{L}}$ and $f_{\mathrm{R}}$ are the kernels fitted for the left and right stimulus regressors, respectively. To add a bilateral input interaction regressor (Model II), the model became $R_{t}=$ $R_{0}+C_{\mathrm{L}, t} * f_{\mathrm{L}}+C_{\mathrm{R}, t} * f_{\mathrm{R}}+C_{\mathrm{L} \cap \mathrm{R}, t} * f_{\mathrm{L} \cap \mathrm{R}}$, where $f_{\mathrm{L} \cap \mathrm{R}}$ is the kernel fitted for the bilateral input interaction regressor. With the addition of an activity history-dependence term, the fitted GLMs became (Model III) $R_{t}=R_{0}+C_{\mathrm{L}, t} * f_{\mathrm{L}}+C_{\mathrm{R}, t} * f_{\mathrm{R}}+R^{\prime}{ }_{t}^{*} f_{\mathrm{h}}$, and (Model IV) $R_{t}=R_{0}+$ $C_{\mathrm{L}, t} * f_{\mathrm{L}}+C_{\mathrm{R}, t} * f_{\mathrm{R}}+C_{\mathrm{L} \cap \mathrm{R}, t} * f_{\mathrm{L} \cap \mathrm{R}}+R^{\prime}{ }_{t} * f_{\mathrm{h}}$, where $R^{\prime}{ }_{t}$ denotes activity history in the preceding 4 frames (i.e., $\left[R_{t-4}, R_{t-3} R_{t-2}, R_{t-1}\right]$ ), and $f_{\mathrm{h}}$ the fitted kernel for the activity history (i.e., modeling up to 2-s preceding activity history-dependence). The kernel sizes for the 1-STIM, r-STIM or b-STIM regressors was 10 (i.e., convolving with stimulus signals in a preceding 5-s window). Coefficients of best fit were determined as those that minimized cross-validation errors. For response prediction using the fitted GLMs, we used an identity link function. Root-mean-square error (RMSE) of each model for each ROI were calculated and compared accordingly. 


\section{References}

Ahrens, M.B., Li, J.M., Orger, M.B., Robson, D.N., Schier, A.F., Engert, F., and Portugues, R. (2012). Brain-wide neuronal dynamics during motor adaptation in zebrafish. Nature 485, 471-477.

Ahrens, M.B., Orger, M.B., Robson, D.N., Li, J.M., and Keller, P.J. (2013). Whole-brain functional imaging at cellular resolution using light-sheet microscopy. Nature Methods 10, 413-420.

Aimon, S., Katsuki, T., Jia, T., Grosenick, L., Broxton, M., Deisseroth, K., Sejnowski, T.J., and Greenspan, R.J. (2019). Fast near-whole-brain imaging in adult Drosophila during responses to stimuli and behavior. Plos Biol 17, e2006732.

Andalman, A.S., Burns, V.M., Lovett-Barron, M., Broxton, M., Poole, B., Yang, S.J., Grosenick, L., Lerner, T.N., Chen, R., Benster, T., et al. (2019). Neuronal Dynamics Regulating Brain and Behavioral State Transitions. Cell 177, 970-985.e20.

Bahl, A., and Engert, F. (2020). Neural circuits for evidence accumulation and decision making in larval zebrafish. Nature Neuroscience 23, 94-102.

Bhattarai, J.P., Schreck, M., Moberly, A.H., Luo, W., and Ma, M. (2020). Aversive Learning Increases Release Probability of Olfactory Sensory Neurons. Curr Biol 30, 31-41.e3. Brand, A., Behrend, O., Marquardt, T., McAlpine, D., and Grothe, B. (2002). Precise inhibition is essential for microsecond interaural time difference coding. Nature 417, 543-547.

Brugge, J.F., Dubrovsky, N.A., Aitkin, L.M., and Anderson, D.J. (1969). Sensitivity of single neurons in auditory cortex of cat to binaural tonal stimulation; effects of varying interaural time and intensity. J Neurophysiol 32, 1005-1024.

Catania, K.C. (2013). Stereo and serial sniffing guide navigation to an odour source in a mammal. Nature Communications 4, 1441.

Chen, T.-W., Wardill, T.J., Sun, Y., Pulver, S.R., Renninger, S.L., Baohan, A., Schreiter, E.R., Kerr, R.A., Orger, M.B., Jayaraman, V., et al. (2013). Ultrasensitive fluorescent proteins for imaging neuronal activity. Nature 499, 295-300.

Chen, X., Mu, Y., Hu, Y., Kuan, A.T., Nikitchenko, M., Randlett, O., Chen, A.B., Gavornik, J.P., Sompolinsky, H., Engert, F., et al. (2018). Brain-wide Organization of Neuronal Activity and Convergent Sensorimotor Transformations in Larval Zebrafish. Neuron 100, 876-890.e5. Chioma, A.L., Bonhoeffer, T., and Hübener, M. (2020). Disparity Sensitivity and Binocular Integration in Mouse Visual Cortex Areas. J Neurosci 40, 8883-8899.

Chivers, D.P., and Smith, R.J.F. (2016). Chemical alarm signalling in aquatic predator-prey systems: A review and prospectus. Écoscience 5, 338-352.

Chronis, N., Zimmer, M., and Bargmann, C.I. (2007). Microfluidics for in vivo imaging of neuronal and behavioral activity in Caenorhabditis elegans. Nat Methods 4, 727-731.

Dieris, M., Ahuja, G., Krishna, V., and Korsching, S.I. (2017). A single identified glomerulus in the zebrafish olfactory bulb carries the high-affinity response to death-associated odor cadaverine. Scientific Reports 7, 40892.

Dragomir, E.I., Štih, V., and Portugues, R. (2020). Evidence accumulation during a sensorimotor decision task revealed by whole-brain imaging. Nat Neurosci 23, 85-93. 
Dunn, T.W., Mu, Y., Narayan, S., Randlett, O., Naumann, E.A., Yang, C.-T., Schier, A.F., Freeman, J., Engert, F., and Ahrens, M.B. (2016). Brain-wide mapping of neural activity controlling zebrafish exploratory locomotion. ELife 5, e12741.

Fehrenbach, J., Weiss, P., and Lorenzo, C. (2012). Variational Algorithms to Remove Stationary Noise: Applications to Microscopy Imaging. IEEE Transactions on Image Processing 21, 4420-4430.

Fernandes, A.M., Mearns, D.S., Donovan, J.C., Larsch, J., Helmbrecht, T.O., Kölsch, Y., Laurell, E., Kawakami, K., Maschio, M. dal, and Baier, H. (2020). Neural circuitry for stimulus selection in the zebrafish visual system. Neuron.

Friedrich, R.W. (2013). Neuronal Computations in the Olfactory System of Zebrafish. Annu Rev Neurosci 36, 383-402.

Gebhardt, C., Auer, T.O., Henriques, P.M., Rajan, G., Duroure, K., Bianco, I.H., and Bene, F. (2019). An interhemispheric neural circuit allowing binocular integration in the optic tectum.

Nat Commun 10, 5471.

Giovannucci, A., Friedrich, J., Gunn, P., Kalfon, J., Brown, B.L., Koay, S.A., Taxidis, J., Najafi, F., Gauthier, J.L., Zhou, P., et al. (2019). CaImAn an open source tool for scalable calcium imaging data analysis. Elife 8, e38173.

Gomez-Marin, A., Duistermars, B.J., Frye, M.A., and Louis, M. (2010). Mechanisms of odor-tracking: multiple sensors for enhanced perception and behavior. Frontiers in Cellular Neuroscience 4, 6.

Haesemeyer, M., Robson, D.N., Li, J.M., Schier, A.F., and Engert, F. (2018). A Brain-wide Circuit Model of Heat-Evoked Swimming Behavior in Larval Zebrafish. Neuron 98, 817-831.e6.

Hagihara, K.M., Ishikawa, A.W., Yoshimura, Y., Tagawa, Y., and Ohki, K. (2020). Long-Range Interhemispheric Projection Neurons Show Biased Response Properties and Fine-Scale Local Subnetworks in Mouse Visual Cortex. Cereb Cortex 31, 1307-1315. Hartman, E.J., and Abrahams, M.V. (2000). Sensory compensation and the detection of predators: the interaction between chemical and visual information. Proc Royal Soc Lond Ser B Biological Sci 267, 571-575.

Hashiguchi, Y., and Nishida, M. (2007). Evolution of Trace Amine-Associated Receptor (TAAR) Gene Family in Vertebrates: Lineage-Specific Expansions and Degradations of a Second Class of Vertebrate Chemosensory Receptors Expressed in the Olfactory Epithelium. Mol Biol Evol 24, 2099-2107.

Heffner, R.S. (2004). Primate hearing from a mammalian perspective. Anatomical Rec Part Discov Mol Cell Evol Biology 281A, 1111-1122.

Heffner, R.S., and Heffner, H.E. (1988). Sound Localization and Use of Binaural Cues by the Gerbil (Meriones unguiculatus ). Behav Neurosci 102, 422-428.

Herrera, K.J., Panier, T., Guggiana-Nilo, D., and Engert, F. (2020). Larval Zebrafish Use Olfactory Detection of Sodium and Chloride to Avoid Salt Water. Curr Biol.

Hubel, D.H., and Wiesel, T.N. (1962). Receptive fields, binocular interaction and functional architecture in the cat's visual cortex. J Physiology 160, 106-154.

Hussain, A., Saraiva, L.R., Ferrero, D.M., Ahuja, G., Krishna, V.S., Liberles, S.D., and 
Korsching, S.I. (2013). High-affinity olfactory receptor for the death-associated odor cadaverine. Proceedings of the National Academy of Sciences 110, 19579-19584. Imig, T.J., and Adrián, H.O. (1977). Binaural columns in the primary field (A1) of cat auditory cortex. Brain Res 138, 241-257.

Kalueff, A.V., Gebhardt, M., Stewart, A.M., Cachat, J.M., Brimmer, M., Chawla, J.S., Craddock, C., Kyzar, E.J., Roth, A., Landsman, S., et al. (2013). Towards a comprehensive catalog of zebrafish behavior 1.0 and beyond. Zebrafish 10, 70-86.

Kara, P., and Boyd, J.D. (2009). A micro-architecture for binocular disparity and ocular dominance in visual cortex. Nature 458, 627-631.

Kawashima, T., Zwart, M.F., Yang, C.-T., Mensh, B.D., and Ahrens, M.B. (2016). The Serotonergic System Tracks the Outcomes of Actions to Mediate Short-Term Motor Learning. Cell 167, 933-946.e20.

Keller, P.J., Schmidt, A.D., Wittbrodt, J., and Stelzer, E. (2008). Reconstruction of Zebrafish Early Embryonic Development by Scanned Light Sheet Microscopy. Science 322, 1065-1069.

Kermen, F., Lal, P., Faturos, N.G., and Yaksi, E. (2020). Interhemispheric connections between olfactory bulbs improve odor detection. Plos Biol 18, e3000701.

Kikuta, S., Kashiwadani, H., and Mori, K. (2008). Compensatory rapid switching of binasal inputs in the olfactory cortex. J Neurosci Official J Soc Neurosci 28, 11989-11997.

Knogler, L.D., Markov, D.A., Dragomir, E.I., Štih, V., and Portugues, R. (2017).

Sensorimotor Representations in Cerebellar Granule Cells in Larval Zebrafish Are Dense, Spatially Organized, and Non-temporally Patterned. Current Biology 27, 1288-1302.

Koide, T., Miyasaka, N., Morimoto, K., Asakawa, K., Urasaki, A., Kawakami, K., and Yoshihara, Y. (2009). Olfactory neural circuitry for attraction to amino acids revealed by transposon-mediated gene trap approach in zebrafish. Proceedings of the National Academy of Sciences of the United States of America 106, 9884-9889.

Koide, T., Yabuki, Y., and Yoshihara, Y. (2018). Terminal Nerve GnRH3 Neurons Mediate Slow Avoidance of Carbon Dioxide in Larval Zebrafish. Cell Reports 22, 1115-1123.

Krishnan, S., Mathuru, A.S., Kibat, C., Rahman, M., Lupton, C.E., Stewart, J., Claridge-Chang, A., Yen, S.-C., and Jesuthasan, S. (2014). The Right Dorsal Habenula Limits Attraction to an Odor in Zebrafish. Current Biology 24, 1167-1175.

Kunst, M., Laurell, E., Mokayes, N., Kramer, A., Kubo, F., Fernandes, A.M., Förster, D., Maschio, M.D., and Baier, H. (2019). A Cellular-Resolution Atlas of the Larval Zebrafish Brain. Neuron 103, 21-38.e5.

Lacoste, A., Schoppik, D., Robson, D.N., Haesemeyer, M., Portugues, R., Li, J.M., Randlett, O., Wee, C.L., Engert, F., and Schier, A.F. (2015). A Convergent and Essential Interneuron Pathway for Mauthner-Cell-Mediated Escapes. Curr Biol 25, 1526-1534.

Lee, P., and Meyers, S. (1996). Chemoattraction and feeding stimulation in crustaceans. Aquaculture Nutrition 2, 157-164.

Lemon, W.C., Pulver, S.R., Höckendorf, B., McDole, K., Branson, K., Freeman, J., and Keller, P.J. (2015). Whole-central nervous system functional imaging in larval Drosophila. Nat Commun 6, 7924. 
Levy, S., and Bargmann, C.I. (2020). An Adaptive-Threshold Mechanism for Odor Sensation and Animal Navigation. Neuron 105, 534-548.e13.

Liang, L., Fratzl, A., Reggiani, J.D.S., Mansour, O.E., Chen, C., and Andermann, M.L. (2020). Retinal Inputs to the Thalamus Are Selectively Gated by Arousal. Curr Biol 30, 3923-3934.e9.

Liberles, S.D. (2015). Trace amine-associated receptors: ligands, neural circuits, and behaviors. Curr Opin Neurobiol 34, 1-7.

Liu, A., Papale, A.E., Hengenius, J., Patel, K., Ermentrout, B., and Urban, N.N. (2020). Mouse Navigation Strategies for Odor Source Localization. Front Neurosci-Switz 14, 218. Longordo, F., To, M.-S., Ikeda, K., and Stuart, G.J. (2013). Sublinear integration underlies binocular processing in primary visual cortex. Nat Neurosci 16, 714-723.

Loring, M.D., Thomson, E.E., and Naumann, E.A. (2020). Whole-brain interactions underlying zebrafish behavior. Curr Opin Neurobiol 65, 88-99.

Louis, M., Huber, T., Benton, R., Sakmar, T.P., and Vosshall, L.B. (2008). Bilateral olfactory sensory input enhances chemotaxis behavior. Nat Neurosci 11, 187-199.

Lovett-Barron, M., Chen, R., Bradbury, S., Andalman, A.S., Wagle, M., Guo, S., and Deisseroth, K. (2020). Multiple convergent hypothalamus-brainstem circuits drive defensive behavior. Nat Neurosci 1-9.

Marques, J.C., Lackner, S., Félix, R., and Orger, M.B. (2018). Structure of the Zebrafish Locomotor Repertoire Revealed with Unsupervised Behavioral Clustering. Curr Biol 28, 181-195.e5.

Martin, H. (1965). Osmotropotaxis in the Honey-Bee. Nature 208, 59-63.

Migault, G., Plas, T.L. van der, Trentesaux, H., Panier, T., Candelier, R., Proville, R., Englitz, B., Debrégeas, G., and Bormuth, V. (2018). Whole-Brain Calcium Imaging during Physiological Vestibular Stimulation in Larval Zebrafish. Curr Biol 28, 3723-3735.e6.

Mills, A.W. (1958). On the Minimum Audible Angle. J Acoust Soc Am 30, 237-246.

Miyasaka, N., Morimoto, K., Tsubokawa, T., Higashijima, S., Okamoto, H., and Yoshihara, Y. (2009). From the Olfactory Bulb to Higher Brain Centers: Genetic Visualization of Secondary Olfactory Pathways in Zebrafish. The Journal of Neuroscience 29, 4756-4767. Miyasaka, N., Arganda-Carreras, I., Wakisaka, N., Masuda, M., Sümbül, U., Seung, S.H., and Yoshihara, Y. (2014). Olfactory projectome in the zebrafish forebrain revealed by genetic single-neuron labelling. Nature Communications 5, ncomms4639.

Moon, Y.-I., Rajagopalan, B., and Lall, U. (1995). Estimation of mutual information using kernel density estimators. Phys Rev E 52, 2318-2321.

Mu, Y., Bennett, D.V., Rubinov, M., Narayan, S., Yang, C.-T., Tanimoto, M., Mensh, B.D., Looger, L.L., and Ahrens, M.B. (2019). Glia Accumulate Evidence that Actions Are Futile and Suppress Unsuccessful Behavior. Cell 178, 27-43.e19.

Mu, Y., Narayan, S., Mensh, B.D., and Ahrens, M.B. (2020). Brain-wide, scale-wide physiology underlying behavioral flexibility in zebrafish. Curr Opin Neurobiol 64, 151-160. Naumann, E.A., Fitzgerald, J.E., Dunn, T.W., Rihel, J., Sompolinsky, H., and Engert, F. (2016). From Whole-Brain Data to Functional Circuit Models: The Zebrafish Optomotor Response. Cell 167, 947-960.e20. 
Oh, S., Harris, J.A., Ng, L., Winslow, B., Cain, N., Mihalas, S., Wang, Q., Lau, C., Kuan, L., Henry, A.M., et al. (2014). A mesoscale connectome of the mouse brain. Nature 508, 207-214.

Ohzawa, I., DeAngelis, G.C., and Freeman, R.D. (1996). Encoding of binocular disparity by simple cells in the cat's visual cortex. J Neurophysiol 75, 1779-1805.

Panier, T., Romano, S.A., Olive, R., Pietri, T., Sumbre, G., Candelier, R., and Debrégeas, G. (2013). Fast functional imaging of multiple brain regions in intact zebrafish larvae using Selective Plane Illumination Microscopy. Front Neural Circuit 7, 65.

Parker, A.J. (2007). Binocular depth perception and the cerebral cortex. Nat Rev Neurosci 8, 379-391.

Pnevmatikakis, E.A., and Giovannucci, A. (2017). NoRMCorre: An online algorithm for piecewise rigid motion correction of calcium imaging data. J Neurosci Meth 291, 83-94. Porter, J., Craven, B., Khan, R.M., Chang, S.-J., Kang, I., Judkewitz, B., Judkewicz, B., Volpe, J., Settles, G., and Sobel, N. (2006). Mechanisms of scent-tracking in humans. Nat Neurosci 10, 27-29.

Portugues, R., Feierstein, C.E., Engert, F., and Orger, M.B. (2014). Whole-Brain Activity Maps Reveal Stereotyped, Distributed Networks for Visuomotor Behavior. Neuron 81, 1328-1343.

Prevedel, R., Yoon, Y.-G., Hoffmann, M., Pak, N., Wetzstein, G., Kato, S., Schrödel, T., Raskar, R., Zimmer, M., Boyden, E.S., et al. (2014). Simultaneous whole-animal 3D imaging of neuronal activity using light-field microscopy. Nature Methods 11, 727-730.

Qian, N. (1997). Binocular Disparity and the Perception of Depth. Neuron 18, 359-368. Rajan, R., Clement, J.P., and Bhalla, U.S. (2006). Rats Smell in Stereo. Science 311, 666-670.

Randlett, O., Wee, C.L., Naumann, E.A., Nnaemeka, O., Schoppik, D., Fitzgerald, J.E., Portugues, R., Lacoste, A.M., Riegler, C., Engert, F., et al. (2015). Whole-brain activity mapping onto a zebrafish brain atlas. Nature Methods 12, 1039-1046.

Reser, D.H., Fishman, Y.I., Arezzo, J.C., and Steinschneider, M. (2000). Binaural Interactions in Primary Auditory Cortex of the Awake Macaque. Cereb Cortex 10, 574-584. Rhodes, M. (1989). Introduction to Particle Technology (Wiley).

Rock, C., and Apicella, A. j (2015). Callosal Projections Drive Neuronal-Specific Responses in the Mouse Auditory Cortex. J Neurosci 35, 6703-6713.

Schindelin, J., Arganda-Carreras, I., Frise, E., Kaynig, V., Longair, M., Pietzsch, T., Preibisch, S., Rueden, C., Saalfeld, S., Schmid, B., et al. (2012). Fiji: an open-source platform for biological-image analysis. Nat Methods 9, 676-682.

Schnupp, J.W.H., and Carr, C.E. (2009). On hearing with more than one ear: lessons from evolution. Nat Neurosci 12, 692-697.

Schnupp, J.W., Mrsic-Flogel, T.D., and King, A.J. (2001). Linear processing of spatial cues in primary auditory cortex. Nature 414, 200-204.

Schröder, S., Steinmetz, N.A., Krumin, M., Pachitariu, M., Rizzi, M., Lagnado, L., Harris, K.D., and Carandini, M. (2020). Arousal Modulates Retinal Output. Neuron 107, 487-495.e9. Sofroniew, N.J., Flickinger, D., King, J., and Svoboda, K. (2016). A large field of view 
two-photon mesoscope with subcellular resolution for in vivo imaging. Elife 5, e14472.

Steck, K., Knaden, M., and Hansson, B.S. (2010). Do desert ants smell the scenery in stereo? Anim Behav 79, 939-945.

Stirman, J.N., Smith, I.T., Kudenov, M.W., and Smith, S.L. (2016). Wide field-of-view, multi-region, two-photon imaging of neuronal activity in the mammalian brain. Nat Biotechnol 34, 857-862.

Suárez, R., Paolino, A., Fenlon, L.R., Morcom, L.R., Kozulin, P., Kurniawan, N.D., and Richards, L.J. (2018). A pan-mammalian map of interhemispheric brain connections predates the evolution of the corpus callosum. Proc National Acad Sci 115, 201808262.

Tsai, P.S., Mateo, C., Field, J.J., Schaffer, C.B., Anderson, M.E., and Kleinfeld, D. (2015).

Ultra-large field-of-view two-photon microscopy. Opt Express 23, 13833-13847.

Vanwalleghem, G., Schuster, K., Taylor, M.A., Favre-Bulle, I.A., and Scott, E.K. (2020).

Brain-Wide Mapping of Water Flow Perception in Zebrafish. J Neurosci 40, 4130-4144.

Vanwalleghem, G.C., Ahrens, M.B., and Scott, E.K. (2018). Integrative whole-brain neuroscience in larval zebrafish. Curr Opin Neurobiol 50, 136-145.

Vladimirov, N., Mu, Y., Kawashima, T., Bennett, D.V., Yang, C.-T., Looger, L.L., Keller, P.J., Freeman, J., and Ahrens, M.B. (2014). Light-sheet functional imaging in fictively behaving zebrafish. Nature Methods 11, 883-884.

Wagner, C.M., Stroud, E.M., and Meckley, T.D. (2011). A deathly odor suggests a new sustainable tool for controlling a costly invasive species. Can J Fish Aquat Sci 68, 1157-1160.

Wakisaka, N., Miyasaka, N., Koide, T., Masuda, M., Hiraki-Kajiyama, T., and Yoshihara, Y. (2017). An Adenosine Receptor for Olfaction in Fish. Curr Biol 27, 1437-1447.e4.

Wee, C.L., Nikitchenko, M., Wang, W.-C.C., Luks-Morgan, S.J., Song, E., Gagnon, J.A., Randlett, O., Bianco, I.H., Lacoste, A.M.B.M., Glushenkova, E., et al. (2019). Zebrafish oxytocin neurons drive nocifensive behavior via brainstem premotor targets. Nature Neuroscience.

Westheimer, G. (1990). Detection of Disparity Motion by the Human Observer. Optometry Vision Sci 67, 627-628.

Wolf, S., Dubreuil, A.M., Bertoni, T., Böhm, U., Bormuth, V., Candelier, R., Karpenko, S., Hildebrand, D.G., Bianco, I.H., Monasson, R., et al. (2017). Sensorimotor computation underlying phototaxis in zebrafish. Nature Communications 8, 651.

Yan, Z., Tan, J., Qin, C., Lu, Y., Ding, C., and Luo, M. (2008). Precise Circuitry Links Bilaterally Symmetric Olfactory Maps. Neuron 58, 613-624.

Yao, Y., Li, X., Zhang, B., Yin, C., Liu, Y., Chen, W., Zeng, S., and Du, J. (2016). Visual Cue-Discriminative Dopaminergic Control of Visuomotor Transformation and Behavior Selection. Neuron 89, 598-612.

Zhang, B., Yao, Y., Zhang, H., Kawakami, K., and Du, J. (2017). Left Habenula Mediates Light-Preference Behavior in Zebrafish via an Asymmetrical Visual Pathway. Neuron 93, 914-928.e4.

Zhang, J., Nakamoto, K.T., and Kitzes, L.M. (2004). Binaural Interaction Revisited in the Cat Primary Auditory Cortex. J Neurophysiol 91, 101-117. 


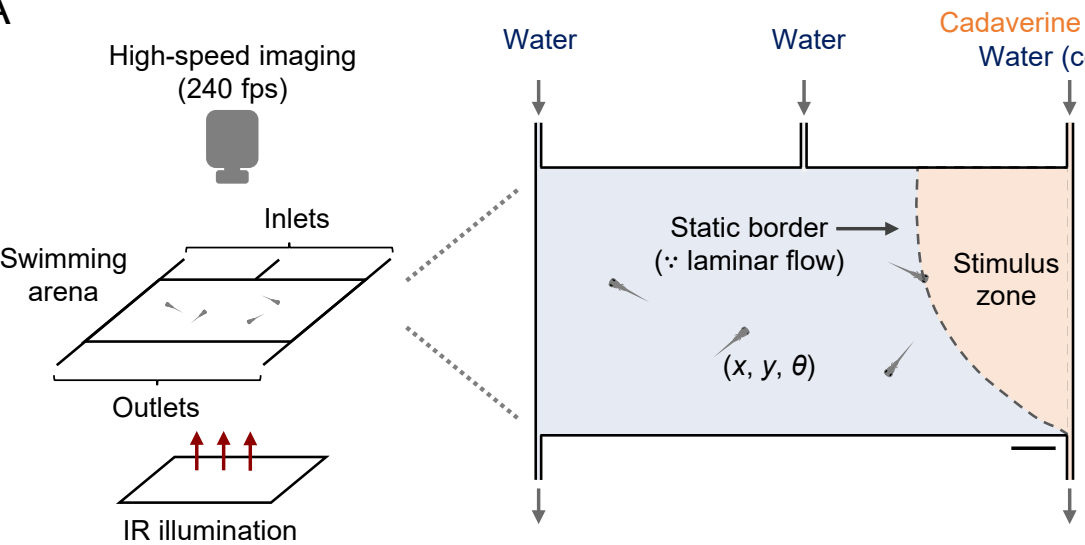

B ater (control)

C

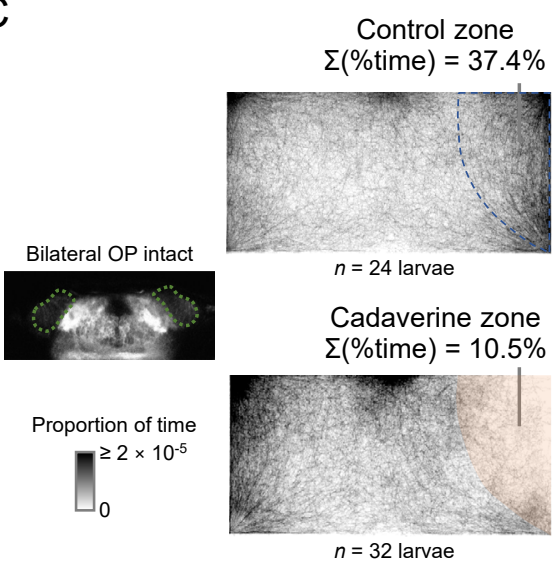

D

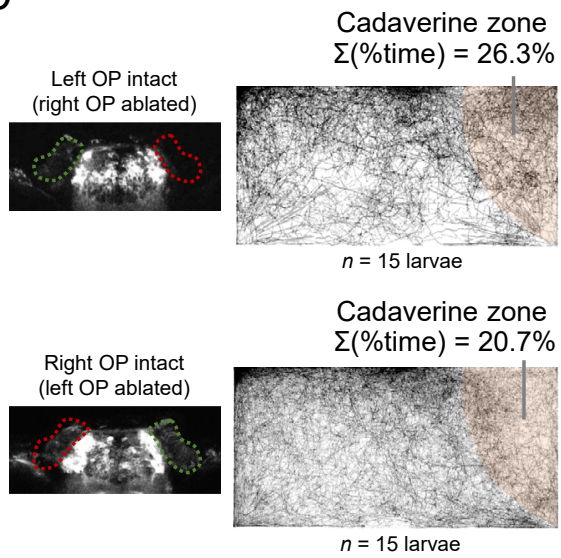

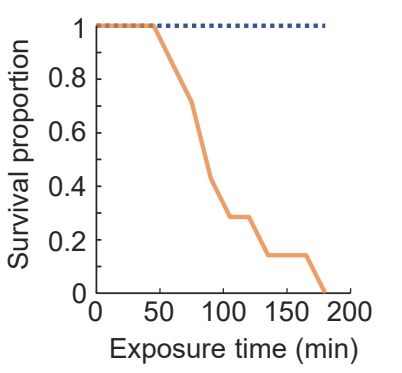

E
F

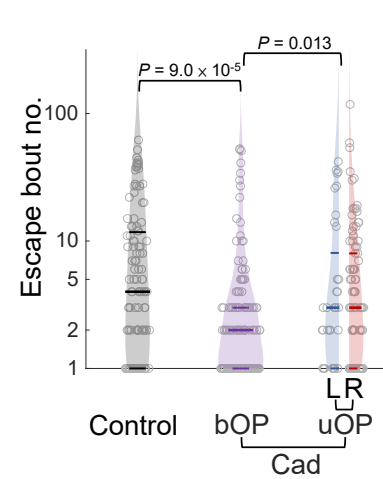

G

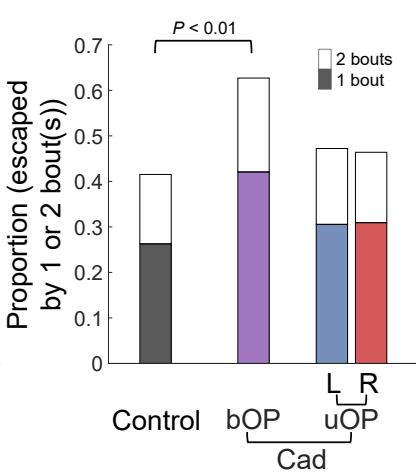

$\mathrm{H}$

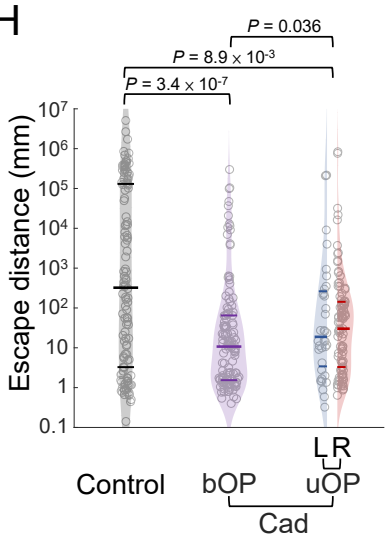

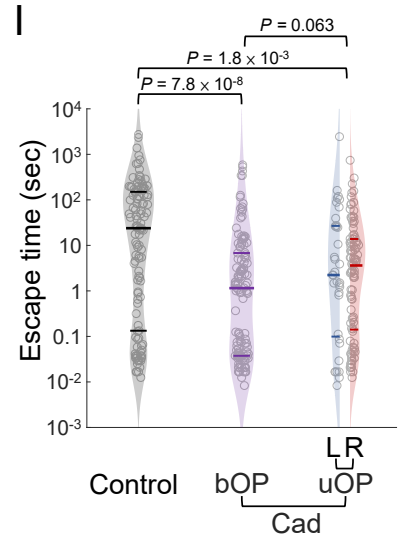

Figure 1. Larval zebrafish performance in a fluidics-based avoidance navigation assay. (A) Schematics of navigation behavioral assay. Zebrafish larvae swimming in a two-dimensional arena $(60 \mathrm{~mm} \times 30 \mathrm{~mm} \times 1.5 \mathrm{~mm})$ are imaged at high-speed (240 fps) under infrared (IR) illumination in the absence of visible light. A noxious chemical zone (stimulus zone) is created and maintained by a constant slow inflow of cadaverine ( $1 \mathrm{mM}$ in water) via the rightmost fluid inlet. Assays in which all zones are filled by water streams serve as control. The laminar flow maintains a static border between the zones. The coordinates $(x, y)$ and orientation $(\theta)$ of the center of the head are tracked and analyzed for each larval zebrafish. Scale bar: $0.5 \mathrm{~cm}$. (B) Proportion of larvae surviving in an arena shown in (A) filled by just cadaverine ( $1 \mathrm{mM}, n=7$, orange solid line) or just water (control, $n=$ 6, blue dotted line) with time. (C) Two-photon image of a zebrafish larva with bilaterally intact olfactory placodes (OPs) (left panel, with OPs outlined by green dotted lines). Footprints of normal zebrafish larvae with bilaterally intact OPs (designated as bOP larvae), expressed in proportion of time occupied by head center coordinates per pixel averaged over time and larvae number, over 2-hour avoidance navigation assays with all zones filled by water streams (right upper panel, with the rightmost zone outlined by dashed line), or the stimulus zone filled by cadaverine stream (right lower panel, with the cadaverine zone highlighted in light orange). (D) Two-photon images of zebrafish larvae with unilateral intact OP (left panels, ablated OPs are outlined by red dotted lines). Footprints of unilateral OP-intact (uOP) larvae, over 2-hour avoidance navigation assays with the stimulus zone filled by cadaverine stream, for the left OP-intact (right upper panel) and right OP-intact (right lower panel) larvae. (E) Illustration of the performance parameters of the avoidance navigation behavior analysed and shown in subsequent plots. (F) Bout number, (G) proportion of entry-to-exit events with only 1 or 2 bouts ( $P$-value: Chi-squared test with Tukey's post-hoc test, comparing 2-bout event proportions), (H) distance travelled, and (I) time taken to escape the stimulus zone for control assays (bOP larvae in water-only arena) and avoidance navigation assays (bOP larvae, left OP-intact (L) or right OP-intact (R) uOP larvae in arenas with cadaverine stream in the stimulus zone). In (F), (H) and (I): Note that the parameters are plotted in $\log$ scales. Horizontal lines indicate the medians, 75 and 25 percentiles for each group. $P$-values: Kruskal-Wallis test with Tukey's post-hoc test. 
A

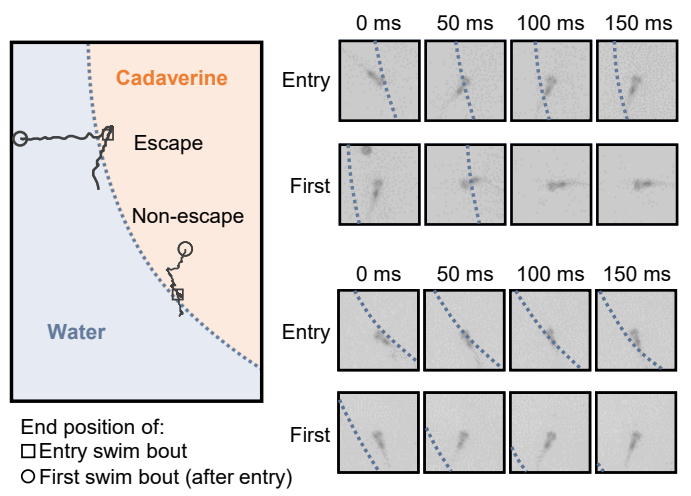

B

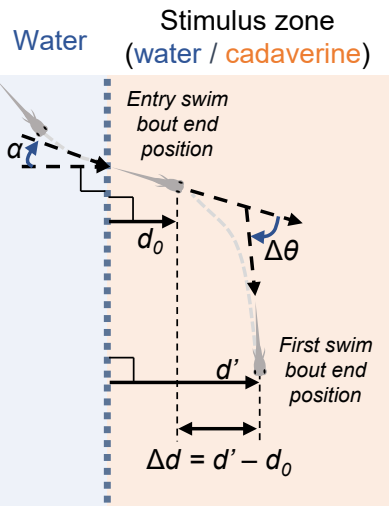

$\mathrm{F}$

$E$

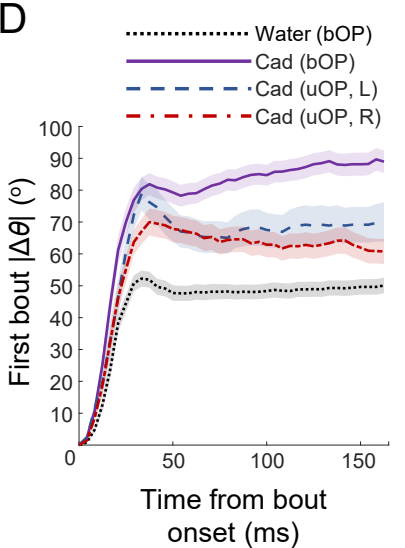

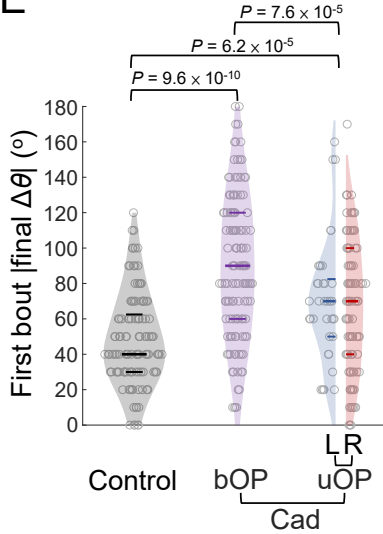

C
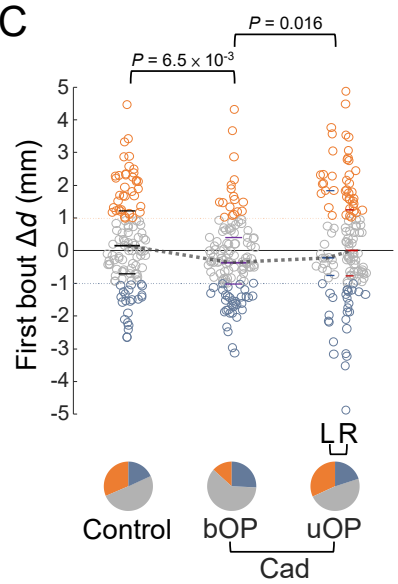

G

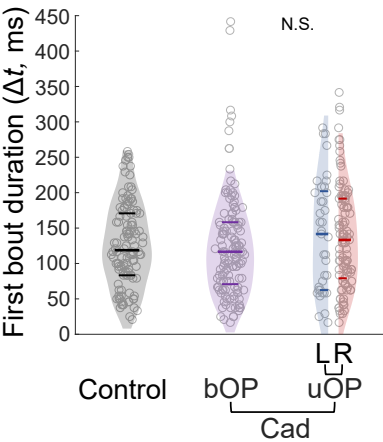

Figure 2. Kinematic parameters of first bouts after entering stimulus zone. (A) Left panel: Two example entry events by a larval zebrafish, whereby the first swim bouts after entering the cadaverine zone resulted in escape (upper example) or further navigation into the region (lower example). Right panel: snapshots of the tracked larval zebrafish at different time points after onset of the corresponding swim bouts. Dotted lines: cadaverine zone border. (B) Schematics of kinematic parameters extracted, including incident angle (relative to vector normal to border) on zone entry $(\alpha)$, change in perpendicular distance (i.e., length of the shortest vector normal to and from the border to the head center) upon first bout completion $(\Delta d)$ and time-dependent change in head orientation $(\Delta \theta)$. (C) - (G) Kinematic parameters of the first bouts, including $(\mathbf{C}) \Delta d$ (medians connected by grey dotted lines), (D) dynamics of $|\Delta \theta|$ after bout onset (line: mean; shadow: SEM), (E) $\mid$ final $\Delta \theta \mid$ on bout completion, (F) average angular velocity (|final $\Delta \theta \mid / \Delta t$ ), and (G) bout duration $(\Delta t)$. | | denotes absolute value. In (C), $\Delta d$ values falling in different ranges are plotted in different colors (orange: $\geq 1 \mathrm{~mm}$, grey: between 1 and $-1 \mathrm{~mm}$, blue: $\leq-1 \mathrm{~mm}$ ). The corresponding proportions of $\Delta d$ in the different ranges for the different groups shown in the pie charts below. For (C) - (G), data are shown for control assays (bilateral OP-intact (bOP) larvae in water-only arena) and avoidance navigation assays (bOP larvae, left (L) or right (R) unilateral OP-intact (uOP) larvae in arena with cadaverine (Cad) in the stimulus zone). In (C), (E) - (G), horizontal lines indicate the medians, 75 and 25 percentiles for each group. $P$-values: Kruskal-Wallis test with Tukey's post-hoc test. N.S.: not significant. 
A
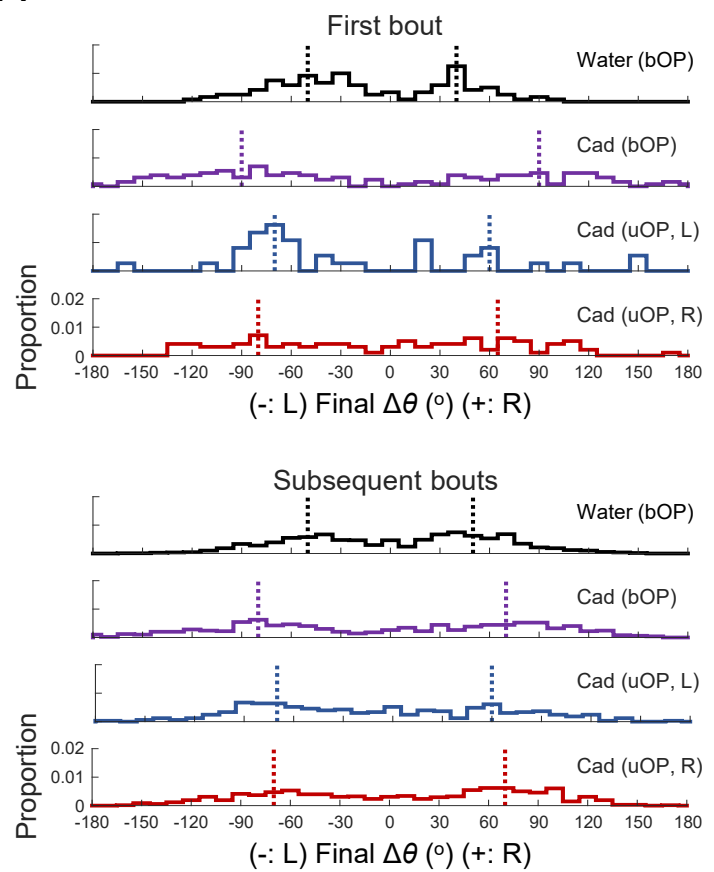

B

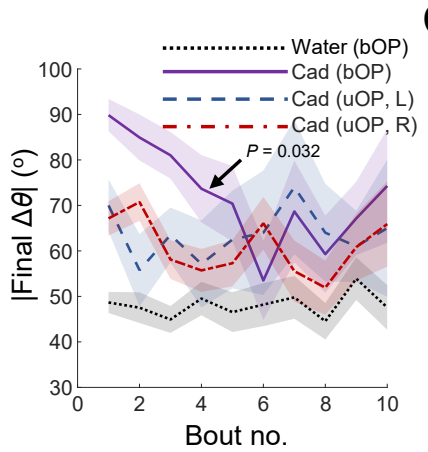

D

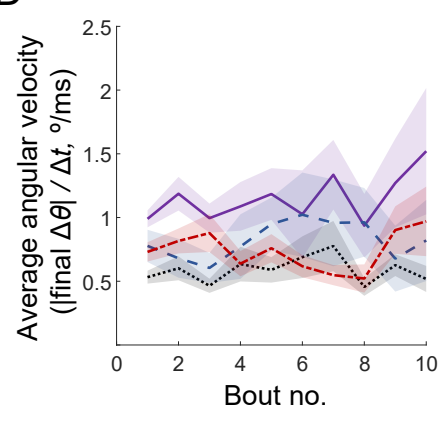

C

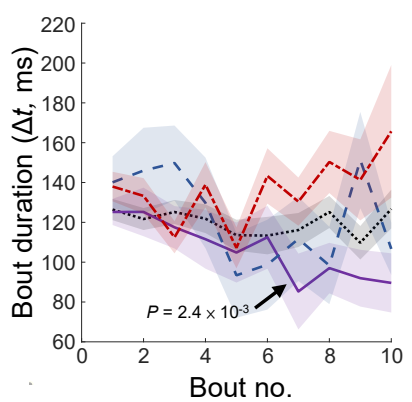

$E$

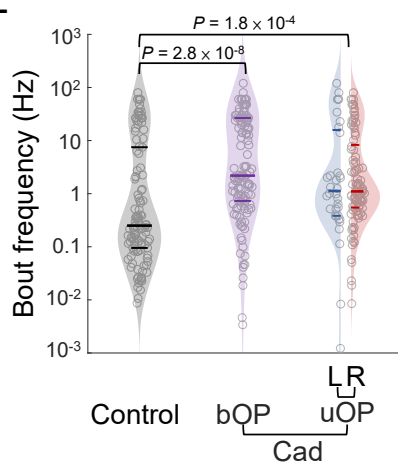

Figure 3. Dependence of kinematic parameters on bout number in the stimulus zone. (A) Histograms of turn angle distributions (i.e., final orientation change on bout completion (final $\Delta \theta$ )) for the first (upper panel) and all subsequent bouts (lower panel) in the stimulus zone for the different experimental groups. Dotted lines indicate the median angles in the corresponding directions. (B) $\mid$ Final $\Delta \theta \mid$, (C) bout duration $(\Delta t)$, and (D) average angular velocity (|final $\Delta \theta \mid / \Delta t$ ) vs. bout number (line: mean; shadow: SEM). $P$-values: Mann-Kendall trend test. | | denotes absolute value. (E) Swim bout frequency in the stimulus zone quantified from all zone entry and escape trajectories (plotted in log scale). $P$-values: Kruskal-Wallis test with Tukey's post-hoc test. In all plots, data are shown for control assays (bilateral OP-intact (bOP) larvae in water-only arena) and avoidance navigation assays (bOP larvae, left $(\mathrm{L})$ or right $(\mathrm{R})$ unilateral OP-intact (uOP) larvae in arena with cadaverine $(\mathrm{Cad})$ in the stimulus zone). In (E), horizontal lines indicate the medians, 75 and 25 percentiles for each group. 


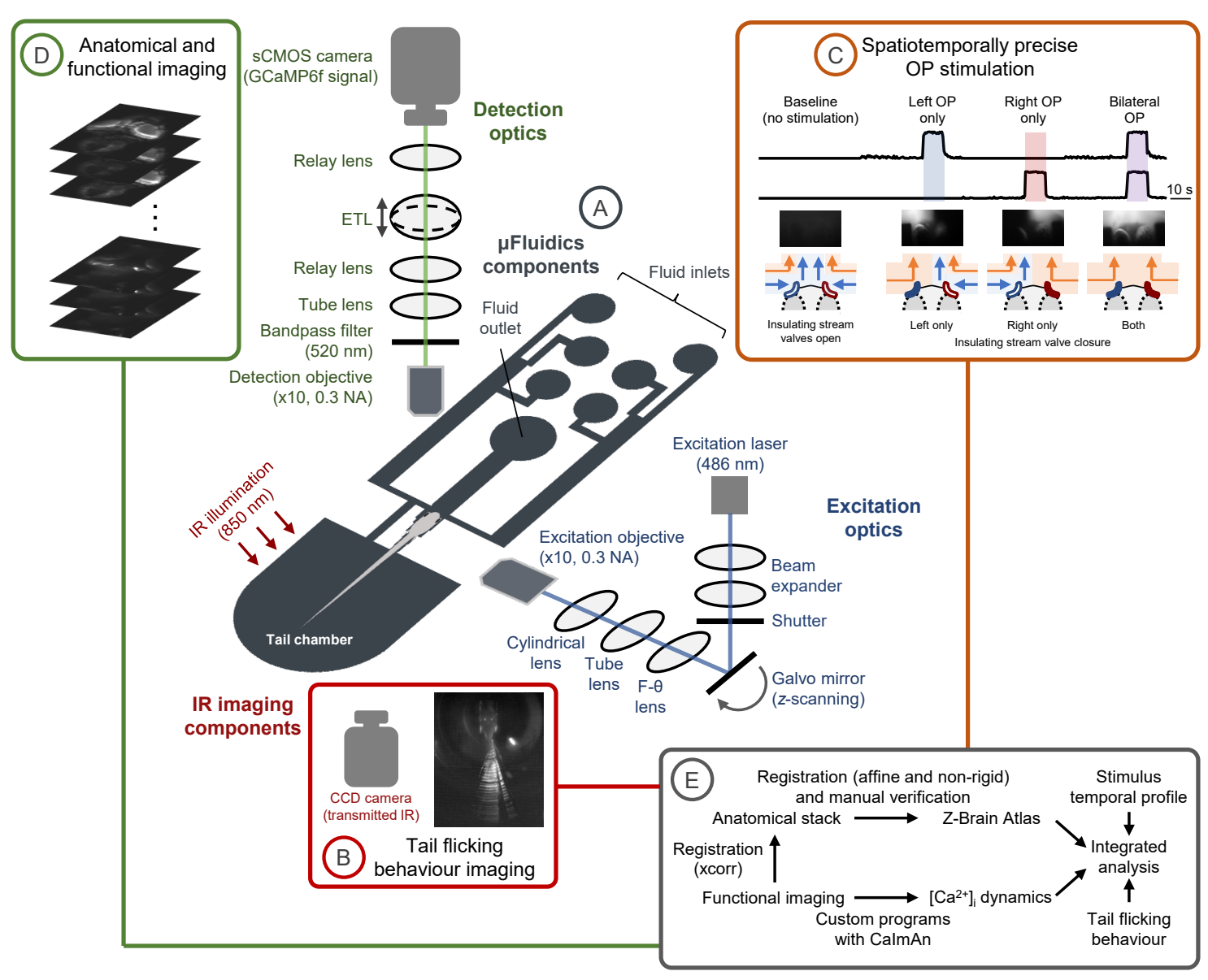

Figure 4. Integrated microfluidics-light sheet microscopy for larval zebrafish whole-brain imaging with spatiotemporally precise olfactory stimulation. (A) In the system, a microfluidic module is integrated with a scanning light sheet microscope. The microfluidic module consists of a PDMS-based device with a tail chamber, a fish head and waist-trapping chamber, fluid inlets connected by tubings and pumps gated by valves for fluid inflow control, and a tube-connected fluid outlet. Also see Supplementary Figure 3A for more detailed drawings and descriptions. (B) To monitor tail flicking behaviour, infrared (IR) illumination and imaging by a CCD camera permits simultaneous motor readout. (C) Spatiotemporally precise olfactory stimulation is achieved by shutting down the water stream(s) immediately in front of the OP(s), which otherwise insulate the cadaverine stream(s) $(1 \mathrm{mM})$, on the left, right, or both sides (illustrated at the bottom row). $1 \mu \mathrm{M}$ fluorescein in the cadaverine stream permits the visualization and monitoring of chemical delivery (upper panels correspond to fluorescence imaging for monitoring the fluid streams, with the readouts shown above). For clarity, the water streams further away to the chemical streams are not shown in this illustration. See Supplementary Figure 3C for more details. (D) On the excitation path, a light sheet is formed by using a cylindrical lens and underfilling the back aperture of the excitation objective, with $z$-scanning achieved by a galvo mirror. On the detection arm of the optics, an electronically tunable lens (ETL) synchronized to the galvo mirror focuses the GCaMP6f signals from the different axial planes of the larval zebrafish brain onto the sCMOS camera. (E) The data analysis pipeline consists of custom-written programs for (i) regional identification in detailed anatomical stack by affine and non-rigid volumetric image registration to the Z-Brain Atlas as the common reference, (ii) calcium dynamics extraction with CaImAn, and regional identification by registration of functional imaging planes to the Z-Brain Atlas-registered anatomical stack, (iii) tail angle extraction, and (iv) integrated analysis with stimulus information (i.e., null, left OP (1-STIM), right OP (r-STIM), or bilateral OP (b-STIM) stimulation) and motor readout. 


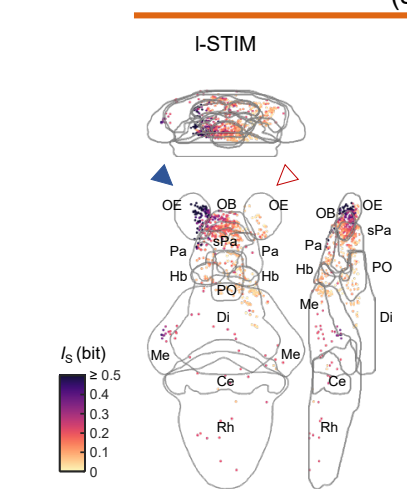

B

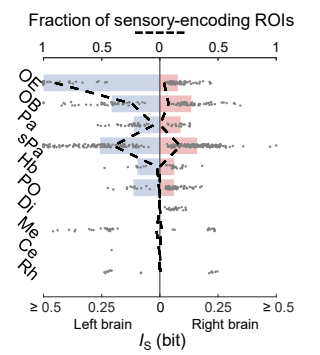

C

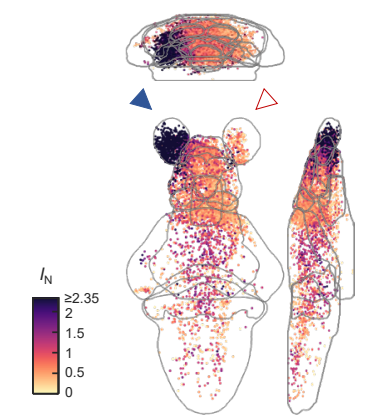

D Mean fraction of sensory-encoding ROIs

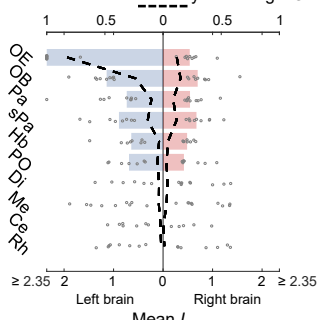

r-STIM

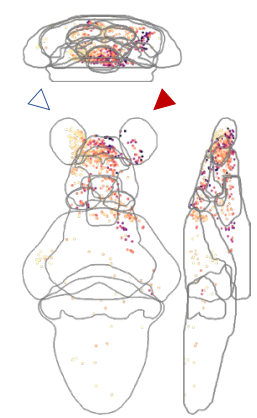

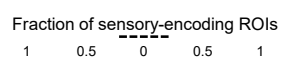
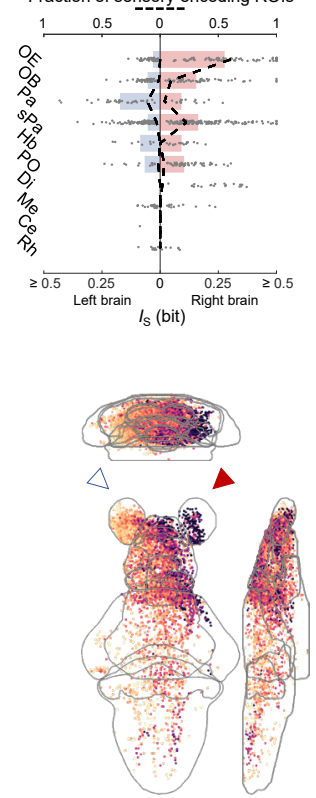

Mean fraction of sensory-encoding ROIs

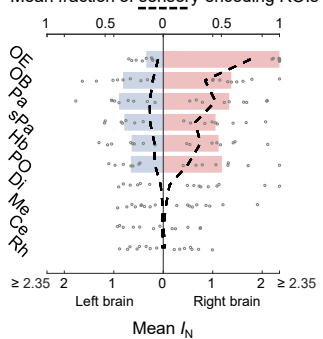

b-STIM

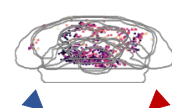

4

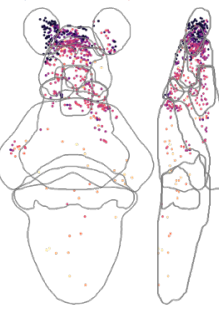

Fraction of sensory-encoding ROIs

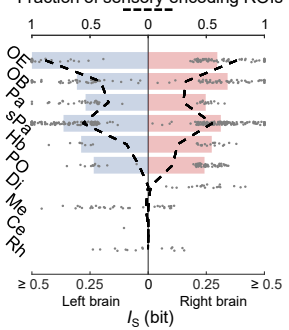

G

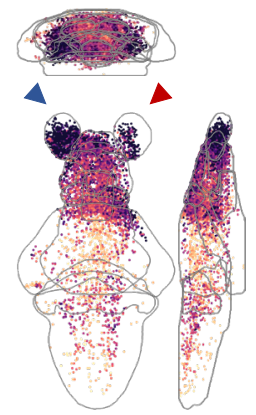

Mean fraction of sensory-encoding ROIs

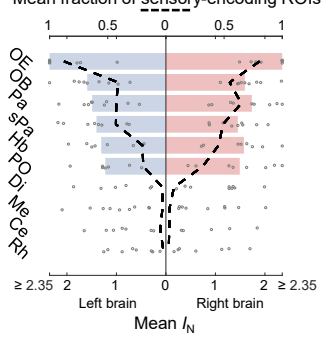

F

$\mathrm{H}$
Motor maps

(motor-encoding neurons)
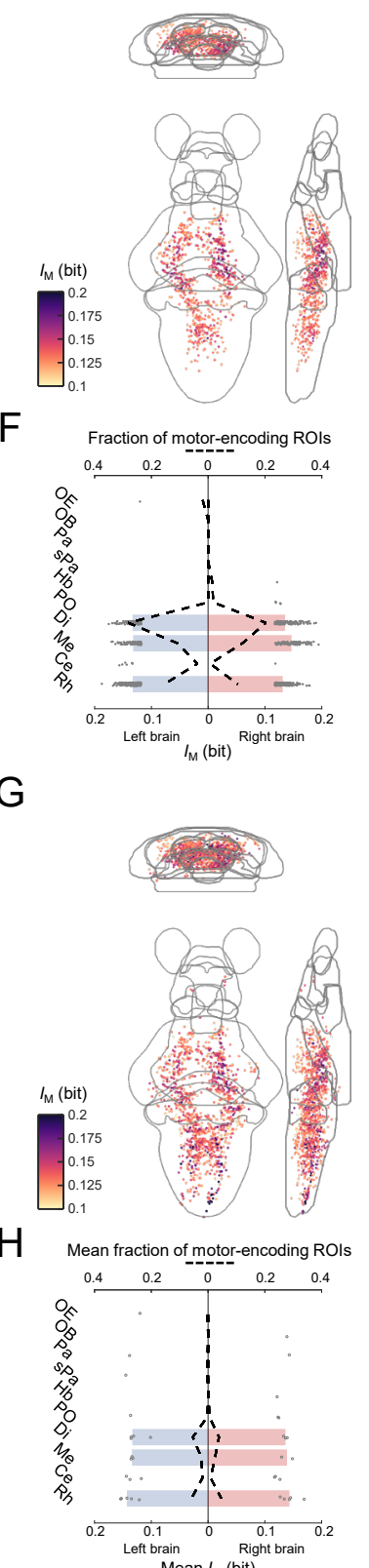

Figure 5. Brainwide sensory- and motor-encoding maps during spatiotemporally precise cadaverine stimulation. (A) Mean intensity projections (to coronal, transverse and sagittal planes) of the mutual information between the calcium signals of regions-of-interest (ROIs) and cadaverine stimulus profile of 1-STIM (left panel), r-STIM (middle panel) or b-STIM (right panel) ( $I_{\mathrm{S}}$ of sensory-encoding ROIs), from an example larval subject. Solid triangles mark the corresponding OP(s) stimulated. Abbreviations: OE, olfactory epithelium; OB, olfactory bulb; Pa, pallium; sPa, subpallium; Hb, habenula; PO, preoptic area; Di, diencephalon; Me, mesencephalon; Ce, cerebellum; Rh, rhombencephalon. (B) From the example fish in (A), the distributions of $I_{\mathrm{S}}$ of sensory-encoding ROIs in the different brain regions with 1-STIM (left panel), r-STIM (middle panel) or b-STIM (right panel). Regions with top six fractions of sensory-encoding ROIs with b-STIM are OE, OB, Pa, sPa, Hb and PO. Bars represent the medians of the $I_{\mathrm{S}}$ of sensory-encoding ROIs in these regions. Dashed lines indicate fractions of sensory-encoding ROIs in all regions. (C) Mean intensity projections of the normalized mutual information between the calcium signals of ROIs and cadaverine stimulus profile of l-STIM (left panel), r-STIM (middle panel) or b-STIM (right panel) pooled across larvae $(n=9$ ) ( $I_{\mathrm{N}}$ of sensory-encoding ROIs). Solid triangles mark the corresponding OP(s) stimulated. (D) The distributions of mean $I_{\mathrm{N}}$ of sensory-encoding ROIs in the different brain regions during l-STIM (left panel), r-STIM (middle panel) or b-STIM (right panel) among the larvae $(n=9)$. Regions with top six mean fractions of sensory-encoding ROIs with b-STIM are OE, OB, Pa, sPa, Hb and PO. Bars represent the medians of the mean $I_{\mathrm{N}}$ of sensory-encoding ROIs in these regions. Dashed lines indicate mean fractions of sensory-encoding ROIs in the different regions among the larvae. (E) Mean intensity projections of mutual information between the calcium signals of ROIs and tail flick frequency ( $I_{\mathrm{M}}$ of motor-encoding ROIs) from the same example larva in (A). (F) The distributions of $I_{\mathrm{M}}$ of motor-encoding ROIs in the different brain regions, from the same example larva in (A). Regions with top three fractions of motor-encoding ROIs are Di, Me and Rh. Bars represent the medians of $I_{\mathrm{M}}$ of motorencoding ROIs in these regions. Dashed lines indicate fractions of motor-encoding ROIs in all regions. (G) Similar to (E) but pooled across larvae $(n=6)$. (H) Similar to $(\mathbf{F})$ but pooled across larvae $(n=6)$, with bars representing the medians of mean $I_{\mathrm{M}}$ of motor-encoding ROIs, and dashed lines indicating mean fractions of motor-encoding ROIs, among the larvae. 


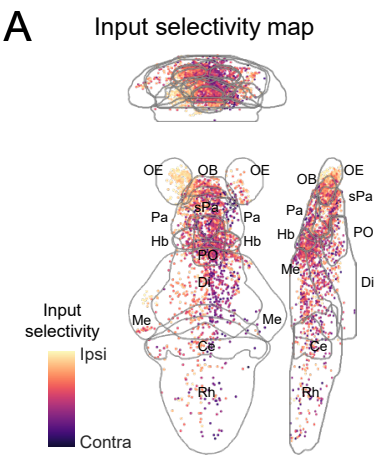

B $\quad$ C

D
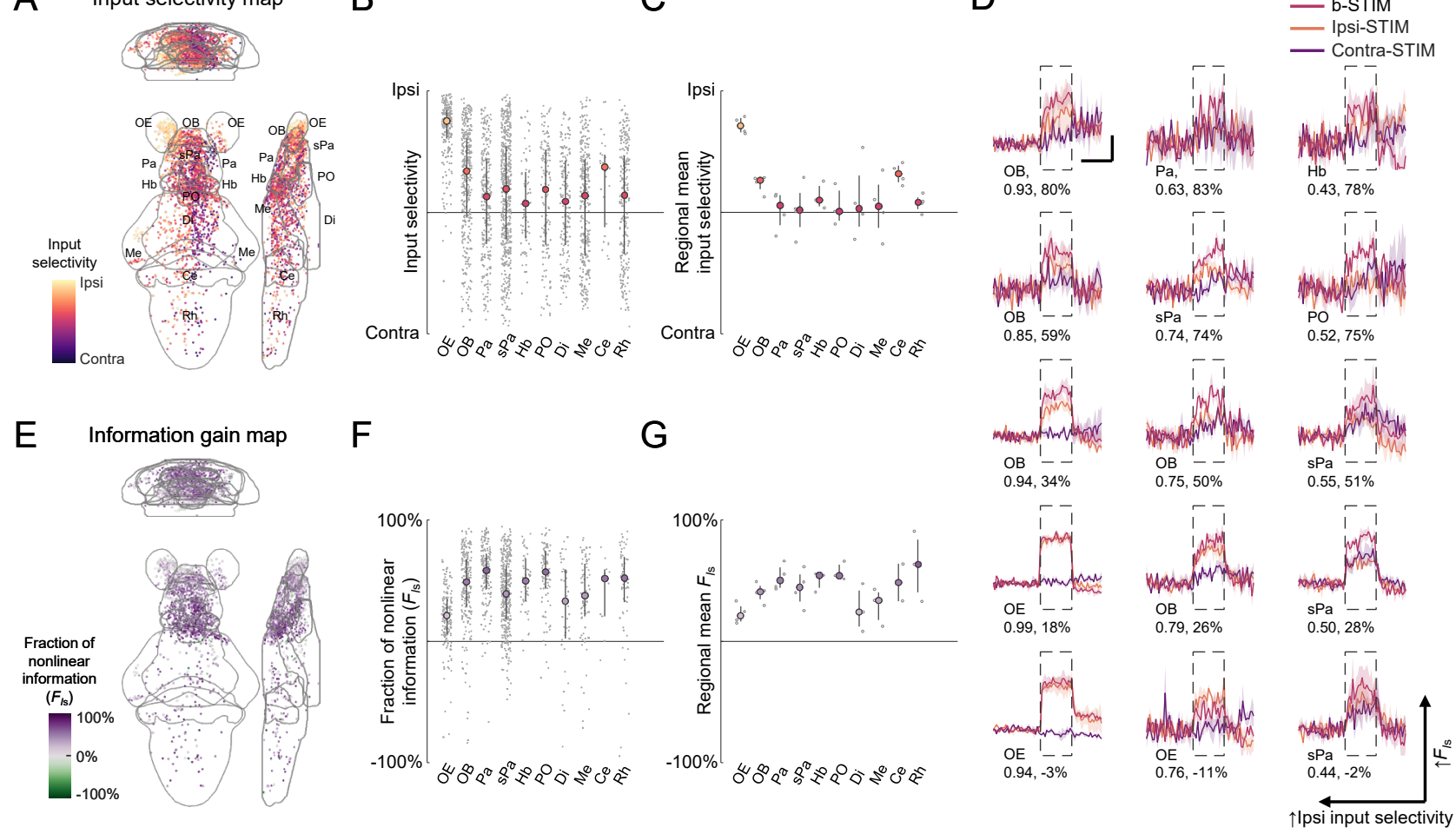

$\mathrm{H}$

I
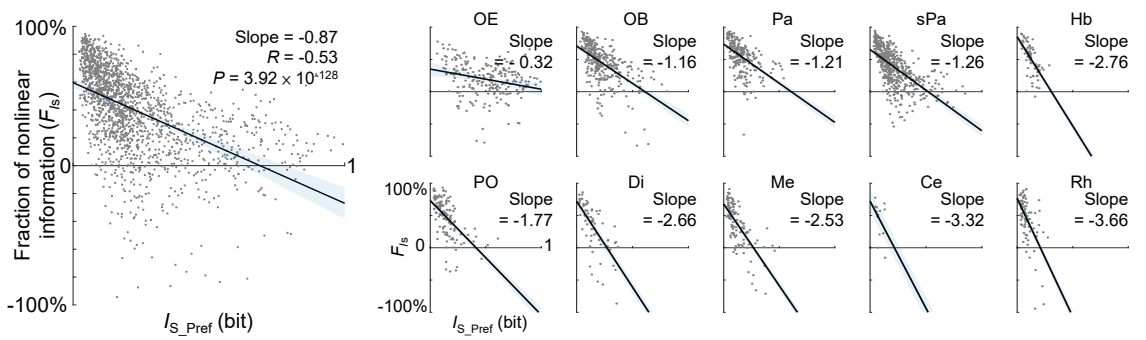

J

Figure 6. Bilateral olfactory input integration properties underlying cadaverine sensing. (A) Mean intensity projection maps (to coronal, transverse and sagittal planes) of ipsilateral(Ipsi)-contralateral(Contra) input selectivity of sensory-encoding regions-of-interest (ROIs) (pooled across larvae, $n=4$ ). Abbreviations: same as in Figure 5. (B) Distributions of the ipsilateralcontralateral input selectivity in the different brain regions. Large dots, upper and lower limits of lines: medians, 75 and 25 percentiles, respectively. (C) Regional means of ipsilateral-contralateral input selectivity, with each small dot representing the value from one larval zebrafish. Large dots, upper and lower limits of lines: medians, 75 and 25 percentiles, respectively. (D) Example trial-averaged responses to ipsilateral (ipsi-STIM, orange), contralateral (contra-STIM, violet) or bilateral (b-STIM, cherry) OP stimulation of individual ROIs from the designated brain regions with a range of ipsilateral-contralateral input selectivity (first number below each example, 1 represents total ipsilateral selectivity, while 0 represents total contralateral selectivity) and fraction of nonlinear information (i.e., fraction of information gained beyond linear summation, denoted by $F_{I S}$ ) (second number below each example). Shadow shows SEM for each trace. Dashed rectangle indicates stimulus window. Scale bars: 10 seconds (horizontal) and 0.5 normalized $d F / F$ (vertical). (E) Mean intensity projection maps of $F_{I \mathrm{~s}}$ (pooled across larvae, $n=4)$ ). (F) Distributions of the $F_{I \mathrm{~s}}$ of individual ROIs in the different brain regions. Large dots, upper and lower limits of lines: medians, 75 and 25 percentiles, respectively. (G) Regional means of $F_{I s}$, with each small dot representing the value from one larval zebrafish. Large dots, upper and lower limits of lines: medians, 75 and 25 percentiles, respectively. (H) $F_{I \mathrm{~S}}$ versus $I_{\mathrm{S}}$ during preferred unilateral OP stimulation by cadaverine $\left(I_{\mathrm{S} \_ \text {Pref }}\right.$, defined as $\max \left(I_{\mathrm{S} \_ \text {-STIM }}, I_{\mathrm{S} \_ \text {r-STIM }}\right)$, where $I_{\mathrm{S} \text { l-STIM }}$ and $I_{\mathrm{S} \text { r-STIM }}$ denote $I_{\mathrm{S}}$ with 1-STIM and r-STIM, respectively) for ROIs pooled across regions and larvae. Black line and light blue shadow show line of best fit on linear regression with $95 \%$ confidence interval. Slope of the line of best fit, correlation coefficient $(R)$ and $P$-values are shown. (I) Similar to $(\mathbf{H})$ but with separate plot and linear regression performed for the ROIs of each brain region. Slopes of the lines of best fit are shown. $(\mathbf{J})$ The absolute values of the slope of $F_{I \mathrm{~s}}$ versus $I_{\mathrm{S} \text { Pref }}$ for each brain region (i.e., |Slope of $F_{I \mathrm{~S}}$ vs. $I_{\mathrm{S} \text { Pref }} \mid$ ), with each small dot representing the value from one larval zebrafish. Large dots, upper and lower limits of lines: medians, 75 and 25 percentiles, respectively. 
A

Sensorimotor functional map

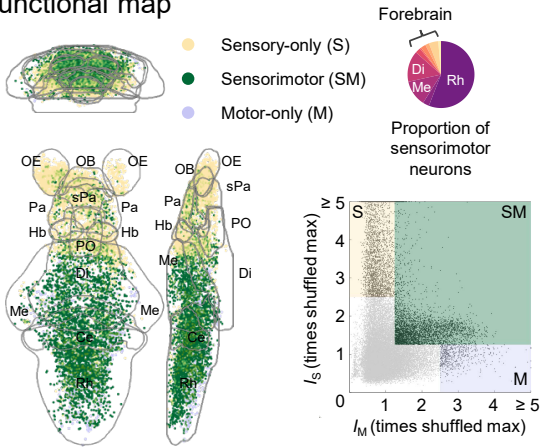

C

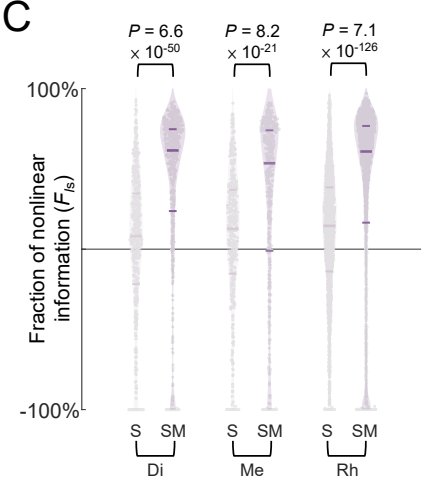

B

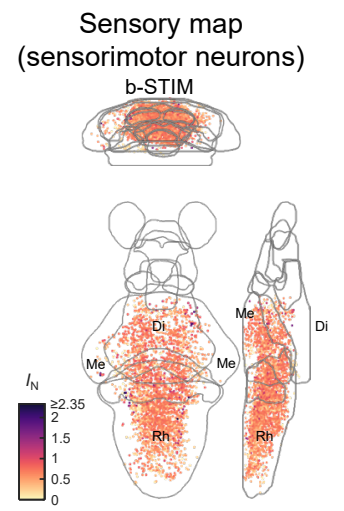

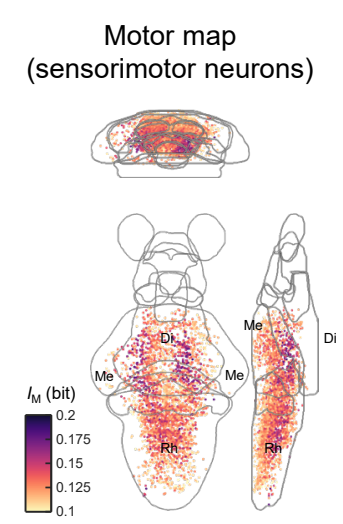

$\mathrm{E}$
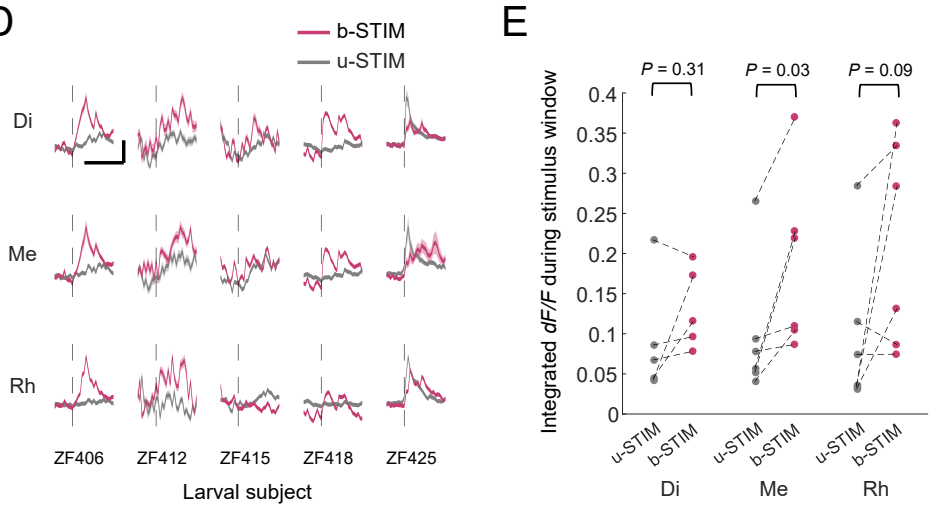

Figure 7. Bilateral olfactory input-dependent activation of sensorimotor units in cadaverine sensing. (A) Left panel: Whole-brain functional maps (projections to coronal, transverse and sagittal planes) of sensory-only (S), sensorimotor (SM) and motor-only (M) ROIs pooled across the behaviorally responsive larvae $(n=6)$. Right upper panel: Pie chart showing the distribution of sensorimotor ROIs in the different brain regions. Right lower panel: Scatter plot showing the distributions of the mutual information between the calcium signals of each ROI and cadaverine stimulus profile (maximum $I_{\mathrm{S}}$ of 1-STIM, r-STIM and b-STIM conditions), and tail flick frequency $\left(I_{\mathrm{M}}\right)$, both calculated as the number of times the maximum of the corresponding shuffled values (see Methods). Abbreviations: Di diencephalon; Me: mesencephalon; Rh: rhombencephalon. (B) Mean intensity projection maps of the mutual information between the calcium signals of sensorimotor ROIs and cadaverine stimulus profile of b-STIM (left panel, normalized mutual information $I_{\mathrm{N}}$ ) and tail flick frequency (right panel, mutual information $I_{\mathrm{M}}$ ), in Di, Me and $\mathrm{Rh}$ pooled across the same group of larvae. (C) Distributions of the fraction of nonlinear information $\left(F_{I s}\right)$ of sensory-only (S) and sensorimotor (SM) ROIs in Di, Me and Rh. Horizontal lines indicate the medians, 75 and 25 percentiles for each brain region. $P$-values: Wilcoxon rank-sum test. (D) Regionally averaged stimulus-locked sensorimotor neuronal responses by bilateral (b-STIM, cherry) and unilateral (u-STIM, grey) cadaverine stimulation from 5 larvae with sensorimotor ROIs identified in all three regions. Vertical dashed line indicates stimulus onset. Scale bars: 10 seconds (horizontal) and 0.5 normalized $d F / F$ (vertical) from each larval subject. Shadows: SEM. (E) Integrated regionally averaged sensorimotor neuronal responses (i.e., summed $d F / F$ over stimulus window) to b-STIM (cherry) and u-STIM (grey). Each pair of connected dots represents data from one larval zebrafish. $P$-values: Wilcoxon signed-rank test. 


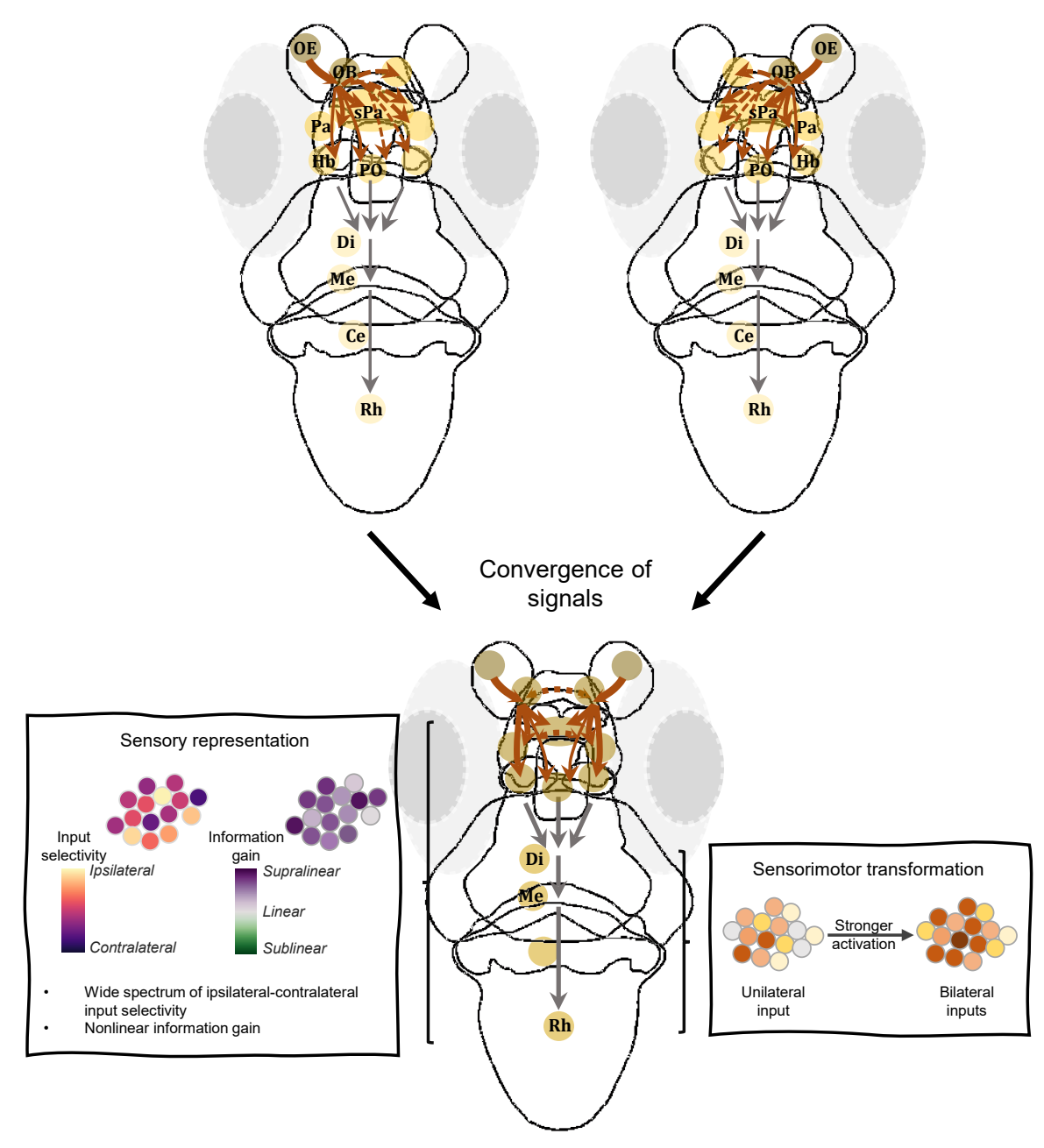

Figure 8. Illustration of brainwide convergence, integration and propagation of olfactory signals in cadaverine sensing. Upper panels: Each olfactory bulb receives inputs from the ipsilateral olfactory epithelium. Upon stimulation, the information is broadcasted to multiple forebrain and downstream brain regions, via ipsilateral and commissural projection pathways. With only unilateral input, the sensory information representation steeply becomes weaker along the rostral-caudal axis. Solid brown arrowed lines represent ipsilateral signal propagation, while dashed brown arrowed lines represent signal propagation to the contralateral side. Lighter colors of circles indicate regions with weaker sensory information representation in cadaverine sensing. Grey lines represent propagation of signals to downstream sensorimotor processing regions. Lower panel: Throughout the olfactory processing pathways, the diversity of stimulus selectivity is preserved. The convergence of signals from the bilateral inputs and their synergistic interactions results in a distributed sensory representation, whereby odor information is especially enhanced (relatively) in the higher-order and caudal brain regions. Meanwhile, this results in stronger activation of sensorimotor neurons. This could be the neural basis of the cadaverine-modulated turn behaviors that exhibit a dependence on bilateral olfactory inputs. 
A

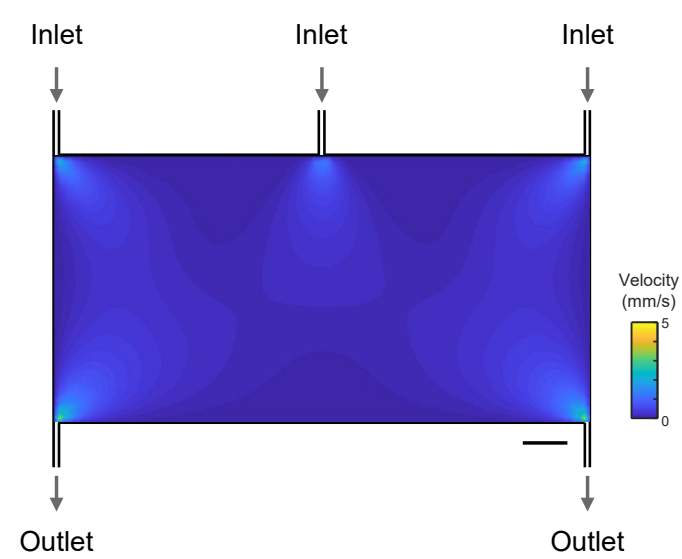

B

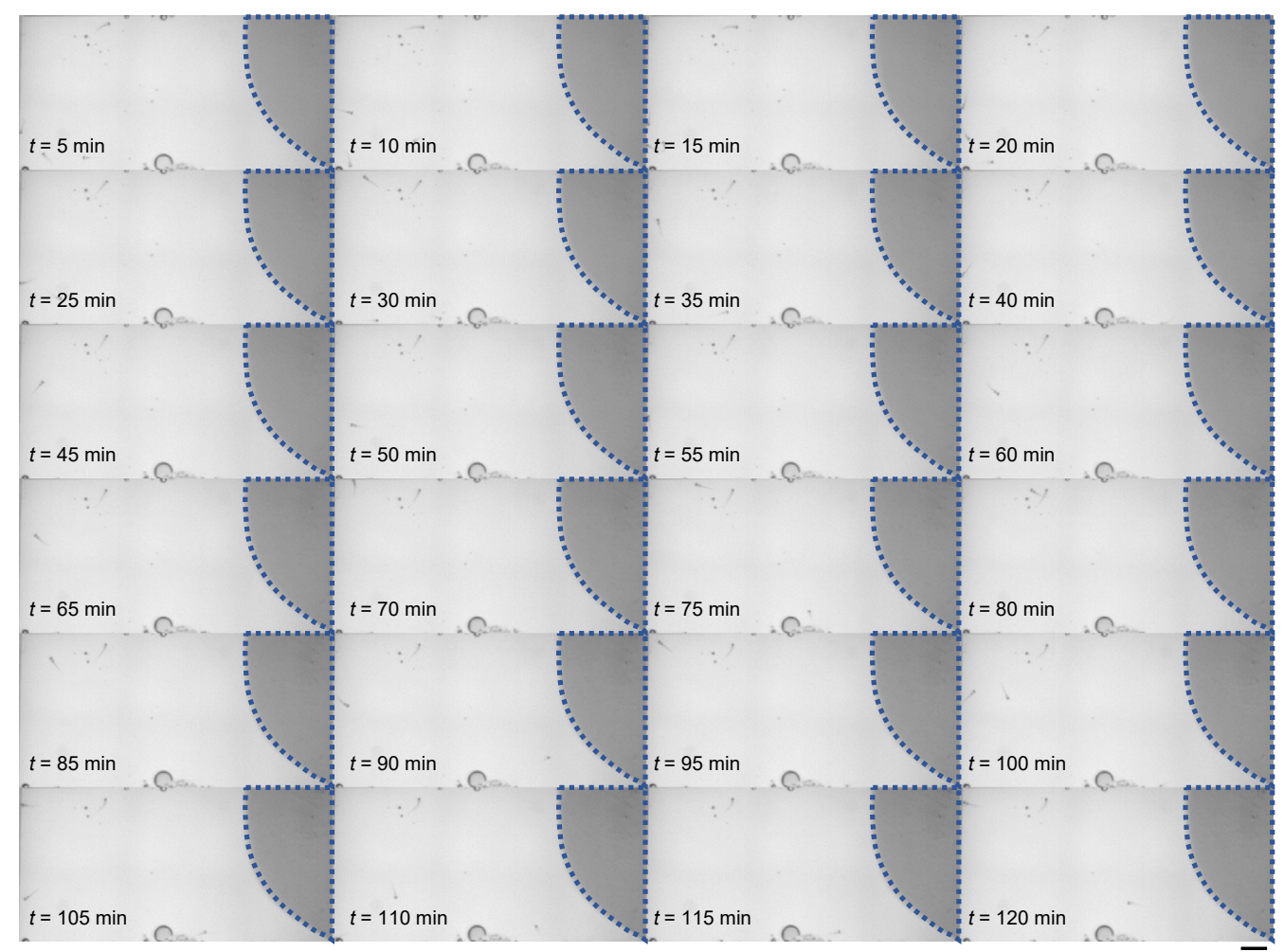

Supplementary Figure 1. Maintenance of a static chemical zone in the fluidics-based avoidance navigation assay. (A) Simulated velocity profile in the swimming arena for navigation assay. (B) Time-lapsed images showing the chemical zone border in the presence of larval zebrafish with an IR dye flowing in via the rightmost fluid inlet in a swimming arena identical to that used for navigation assay. Dotted lines in each image outline the same border. Scale bars in (A) and (B): $0.5 \mathrm{~cm}$. 
A

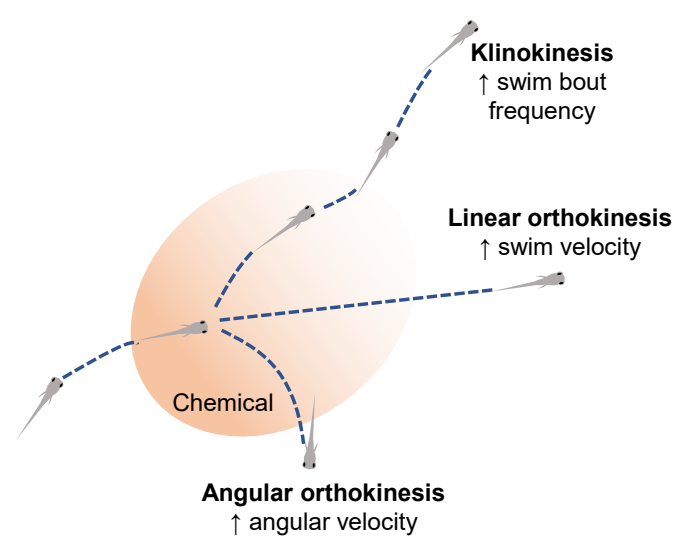

$\mathrm{D}$

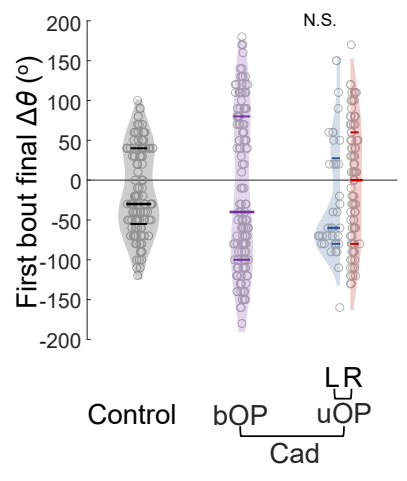

B

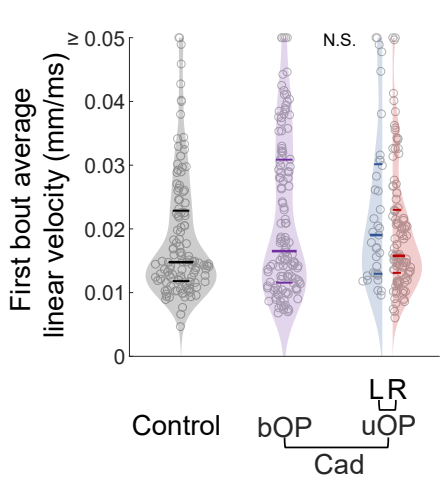

$E$
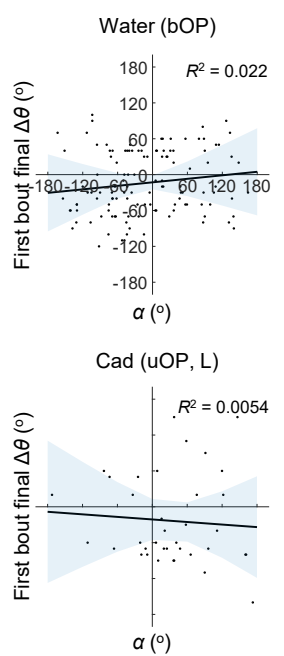
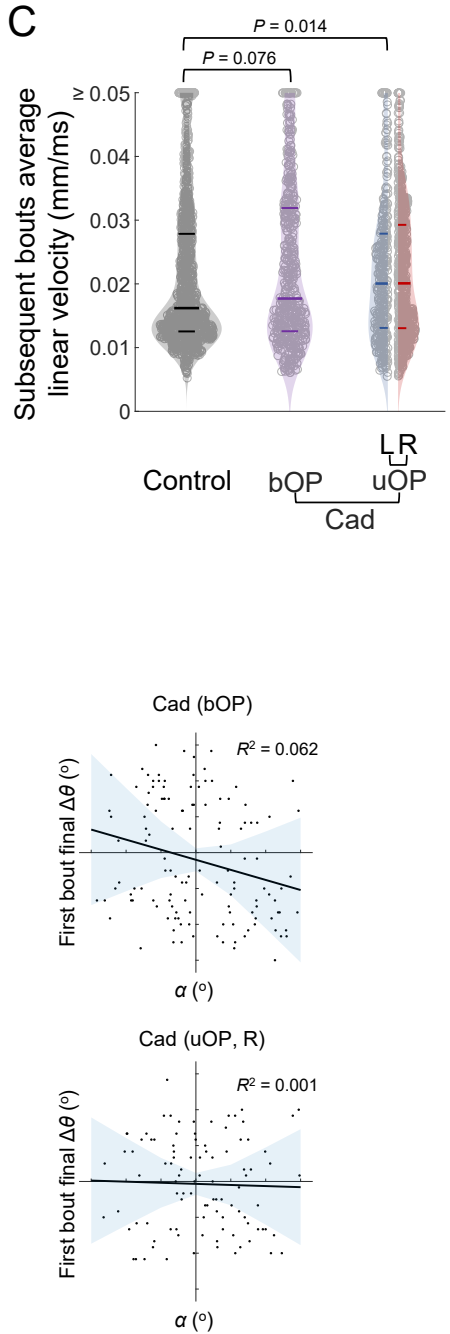

Supplementary Figure 2. Kinematic parameters of first and subsequent bouts after entering cadaverine zone. (A) Illustration of navigational strategies that may be adopted after encountering a noxious chemical. (B) and (C) Distributions of average linear velocity of post-cadaverine zone-entry first (B) and subsequent (C) bouts. $P$-values: Kruskal-Wallis test with Tukey's post-hoc test. N.S.: non-significant. (D) Final $\Delta \theta$ for the first bouts. In (B) - (D), horizontal lines indicate the medians, 75 and 25 percentiles for each group. (E) First turn final $\Delta \theta$ plotted against incidence angle $(\alpha)$ on zone entry (also see Figure 2B). Coefficients of determination $\left(R^{2}\right)$ are shown. Lines and shadows: linear regression with $95 \%$ confidence intervals. In (D) and (E), $\Delta \theta<0$ and $>0$ correspond to left and right turns, respectively. Sign of $\alpha$ follows the same convention as that of $\Delta \theta$. 


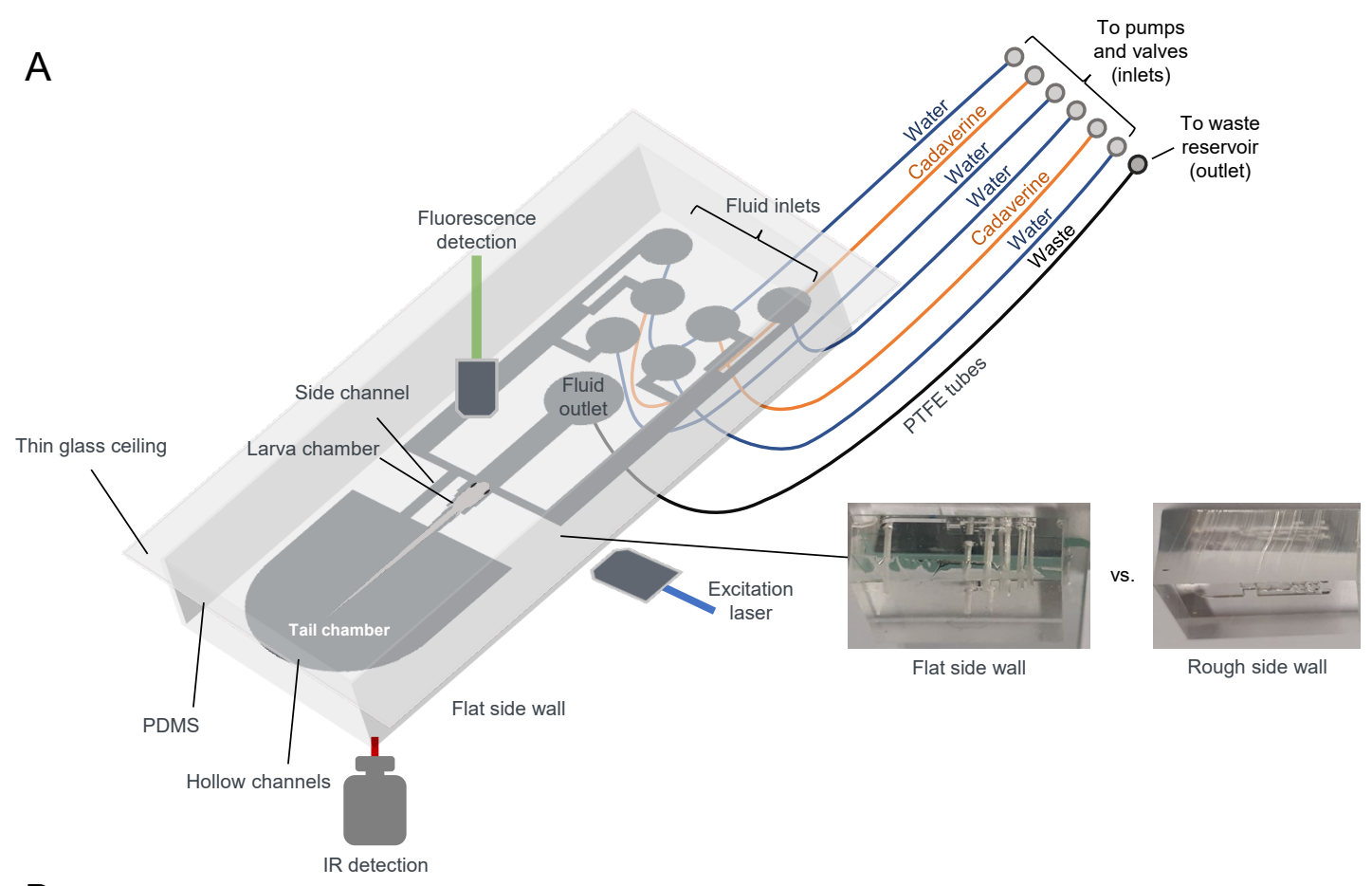

B

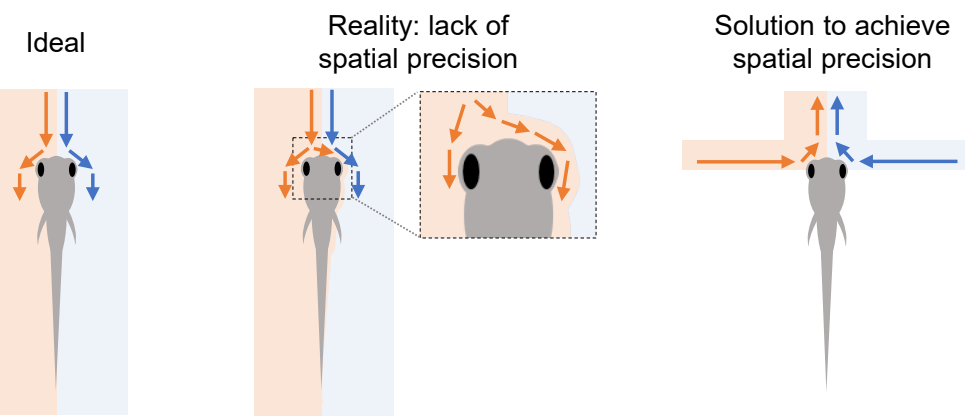

C

Solution to achieve spatiotemporal precision
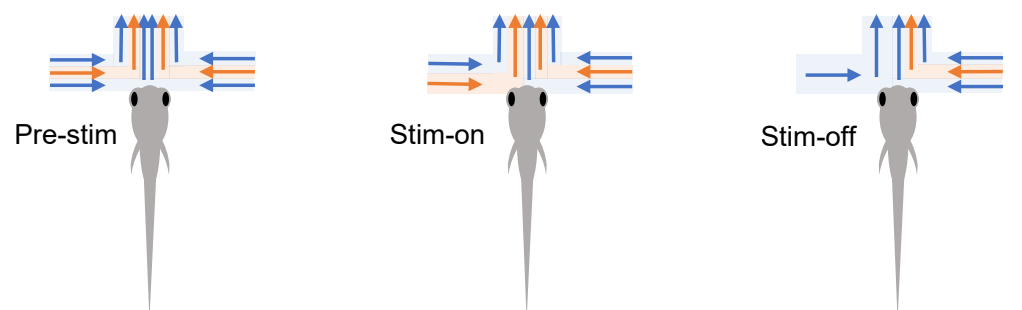

Supplementary Figure 3. Detailed schematics of the microfluidic device for olfactory stimulation and whole-brain imaging, and principles of achieving spatiotemporally precise olfactory stimulation. (A) Detailed schematics of the PDMSbased microfluidic device, that is compatible with (i) the perpendicular orientations of the excitation and detection optics of the custom-built light sheet microscope for whole-brain calcium imaging, and (ii) IR imaging module for the monitoring of stimulus-associated tail flicking behavior. The thin glass ceiling and the flat side wall (see photos for comparison, also see Methods) ensure undisturbed light propagation along the detection and excitation arms, respectively. Fluids are carried into and away from the microfluidic device via PTFE tubes, with inlets connected to valves and syringe pumps, and outlet to a waste reservoir. A long working distance detection objective (Nikon Plan Fluorite, $\times 10$, N.A. 0.3, $16 \mathrm{~mm}$ WD) was used to accommodate the setup. (B) Left panel: Schematic depiction of the ideal segregation of different fluidic streams delivered from the front for unilateral OP stimulation with spatial precision. Light blue: water stream. Light orange: chemical stream. Arrows indicate the flow directions of the fluidic streams. Middle panel: In practical tests, we encountered unavoidable mixing of the fluidic streams flowing parallel from the front towards the larval zebrafish subject. This caused undesired spillover of chemical stimulus to the contralateral OP. Right panel: The problem was solved by directing fluidic streams from the sides to the OPs. The opposite directions of the streams that converge at the midline provided the necessary opposing forces to prevent spillover and achieve the required spatial precision of chemical stimulation. (C) The layout of fluidic streams that was eventually implemented to permit spatiotemporally precise chemical stimulation of unilateral OP. Left panel: At baseline (Pre-stim), the laminar water streams insulate the OPs from the chemical streams. Middle panel: By valve closure, the insulating water stream is removed (left side illustrated), and the chemical stream reaches the unilateral OP with a rapid rise time (Stim-on). Right panel: When the chemical stream is removed by valve closure, only the remaining water stream reaches the OP (Stim-off). Bilateral stimulation is achieved by undergoing the same sequence of fluid stream changes on both sides. 
A

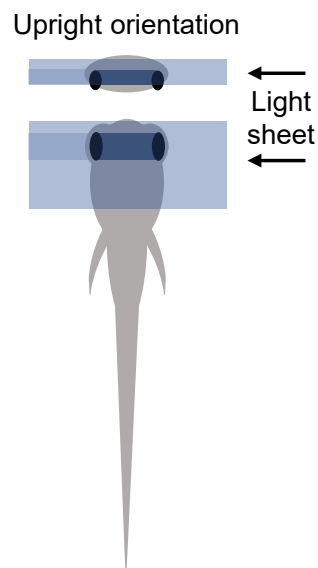

B

Right eye-ablated

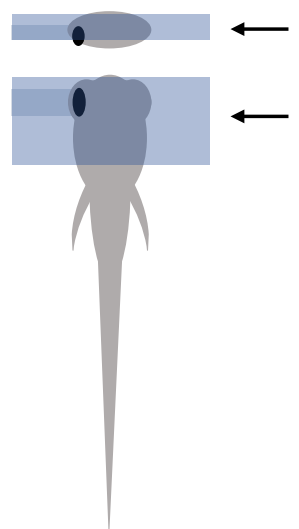

C

Right-tilted orientation

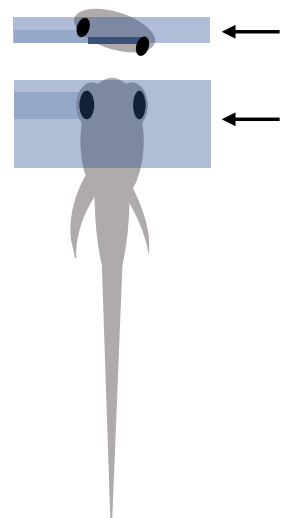

D
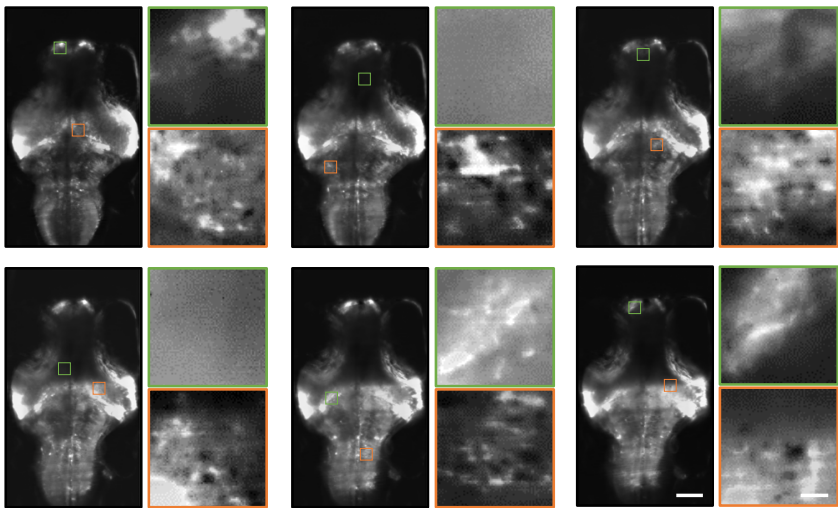

E
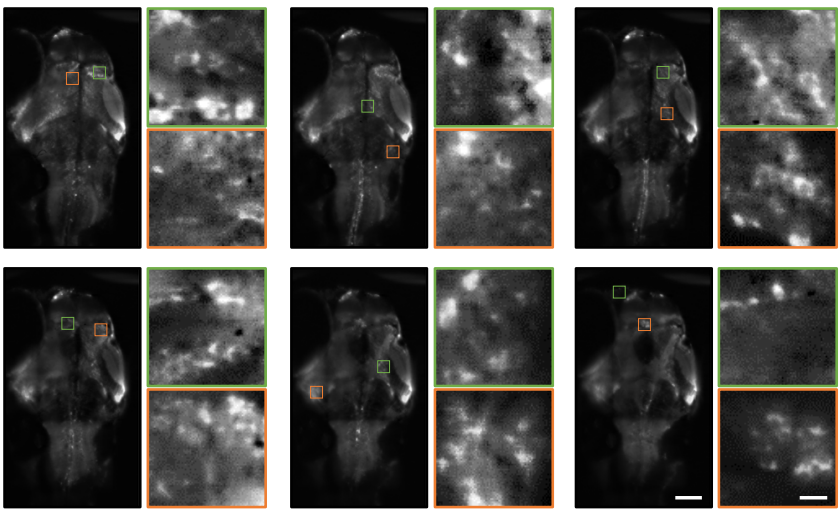

$\mathrm{F}$
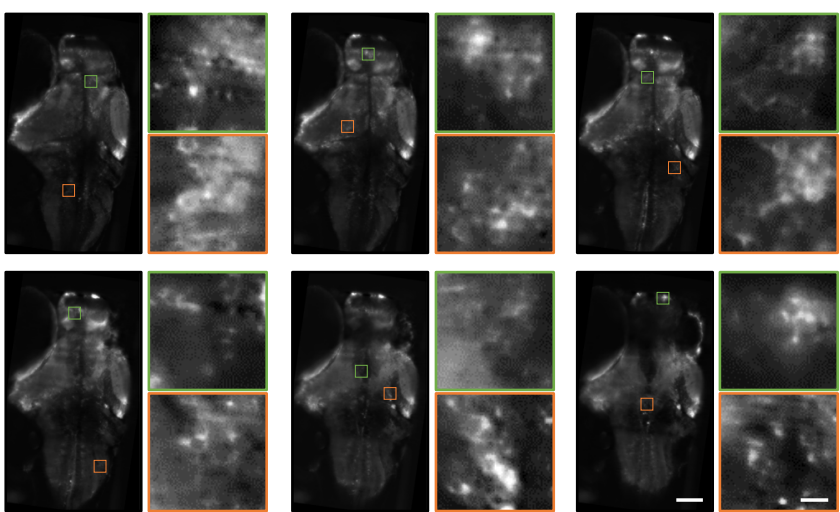

Supplementary Figure 4. Illustration of the different physical configurations of larval zebrafish subjects under imaging. (A) With an upright orientation, the right eye occludes the light sheet and results in poor illumination of the brain regions between the eyes. (B) Imaging after surgical ablation of the right eye (12-24 hours prior to imaging), or (C) tilting by up to $20^{\circ}$ to the right, circumvent the occlusion of light sheet entry by the right eye, thereby avoiding or minimizing poorly imaged regions. Upper panels: view from the tail of the larva. Lower panels: top view. Blue shadow represents the incoming light sheet with the direction indicated by the black arrows (i.e., from right to left). Areas with poor excitation are highlighted in darker colors. (D) - (F) Example images from different planes in three larval zebrafish subjects imaged in (D) eyes intact and upright, (E) right eye-ablated, and (F) right-tilted configurations, respectively. For each image set, the two images on the right are enlarged and contrast-adjusted images from the marked areas of the corresponding large field-of-view (FOV) images. Scale bars (white): $100 \mu \mathrm{m}$ for the large FOV images and $10 \mu \mathrm{m}$ for the zoomed-in images. 
A

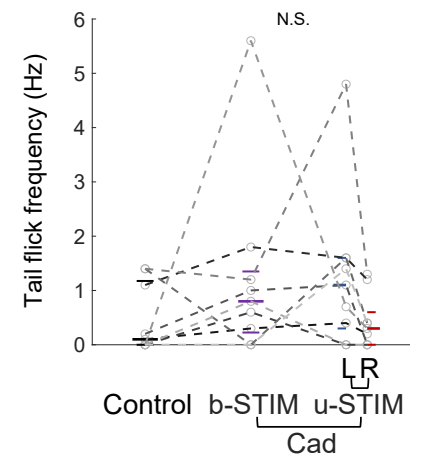

C

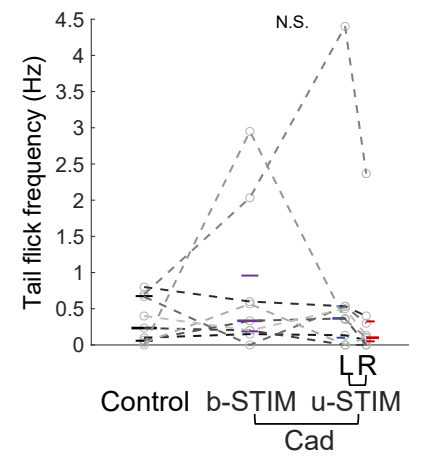

B

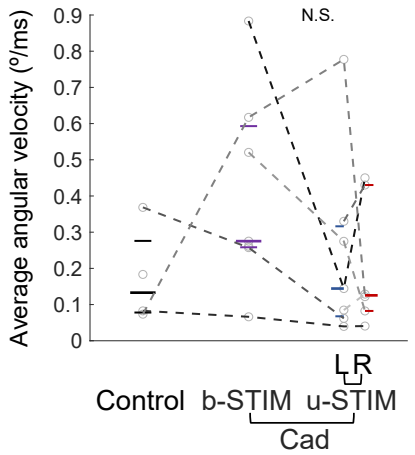

D

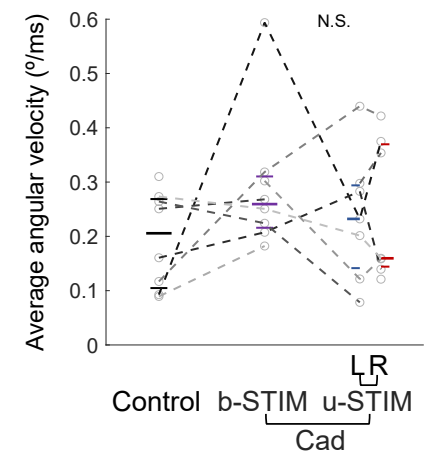

Supplementary Figure 5. Larval zebrafish tail flicking response under restrained imaging. (A) Tail flick frequency and (B) first-bout average angular velocity of all behaviorally responsive larvae $(n=9)$ in the first trials of each stimulus condition. In all plots, one dot represents the mean of a given parameter of a larval subject in each stimulus condition. Data points from the same larval subjects are connected by dashed lines. Horizontal lines indicate the medians, 75 and 25 percentiles for each condition. P-values: Kruskal-Wallis test. N.S.: not significant. (C) and (D), similar to (A) and (B) respectively but with all bouts of all trials of each stimulus condition included. 
A

(sensory-encoding neurons)

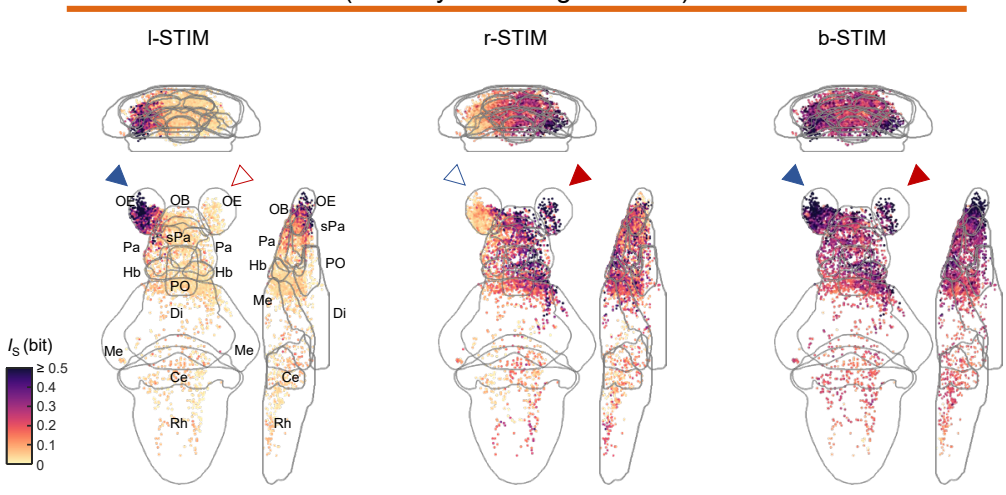

B

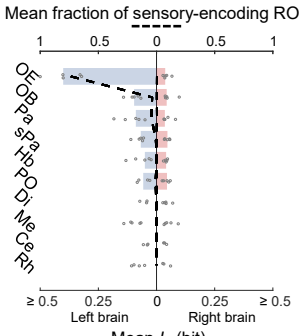

C

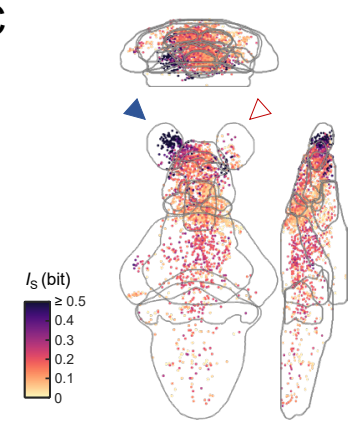

D

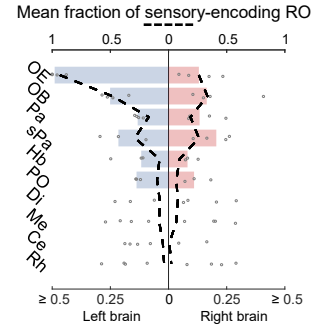

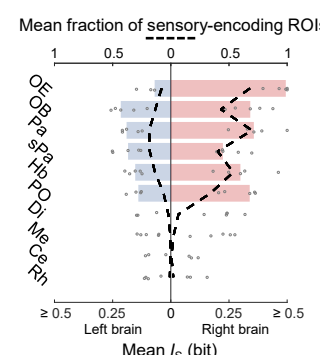

Mean $I_{S}$ (bit)

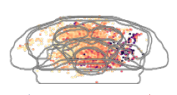

$\Delta$
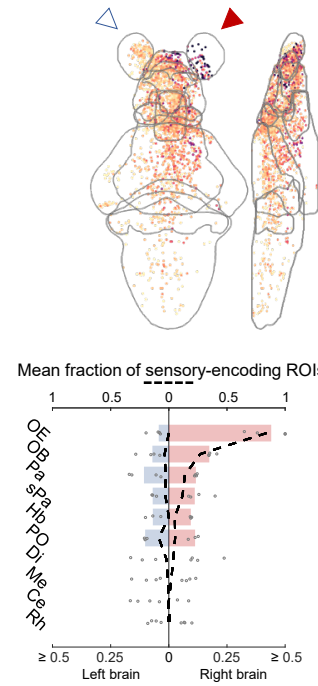

Mean $I_{S}$ (bit)

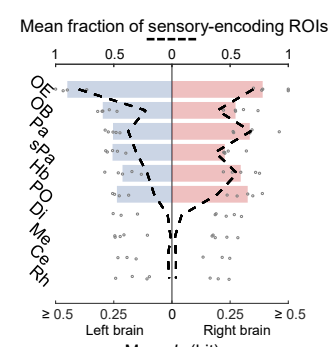

Mean $I_{S}$ (bit)
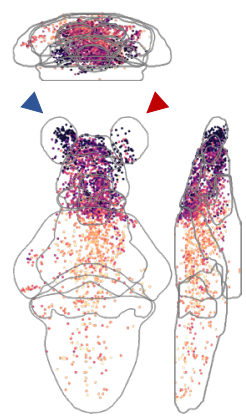

Mean fraction of sensory-encoding ROIs

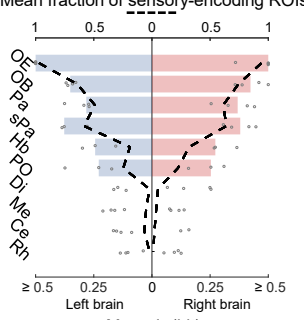

Mean $I_{S}$ (bit)

Supplementary Figure 6. Brainwide activation maps of the larval zebrafish subgroups with biased or unbiased responses during spatiotemporally precise cadaverine stimulation. (A) Mutual information $\left(I_{\mathrm{S}}\right)$ maps pooled across 5 right eye-ablated larvae with biased responses (see Methods). Mean intensity projections of $I_{\mathrm{S}}$ between the calcium signals of sensory-encoding ROIs and cadaverine stimulus profile of l-STIM (left panel), r-STIM (middle panel) or b-STIM (right panel) are shown. Solid triangles mark the corresponding OP(s) stimulated. Abbreviations: same as in Figure 4. (B) The distributions of mean $I_{\mathrm{S}}$ of sensory-encoding ROIs in the different brain regions during l-STIM (left panel), r-STIM (middle panel) or b-STIM (right panel) among the larvae $(n=5)$. Regions with top six mean fraction of sensory-encoding ROIs with b-STIM are OE, OB, Pa, sPa, Hb and PO. Bars representing the medians of the mean $I_{\mathrm{S}}$ of sensory-encoding ROIs in these regions. Dashed lines indicate fractions of sensory-encoding ROIs in the different regions among the larvae. (C) and (D) Similar to (A) and (B) but with data pooled across larvae with generally unbiased responses to 1-STIM or r-STIM ( $n=4$, consisting of 3 right-tilted and 1 right eye-ablated larvae, see Methods). 
Model I (without interaction regressor)

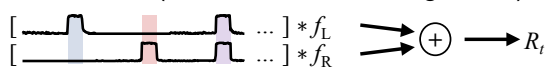

Model II (with interaction regressor)

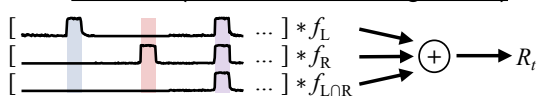

C

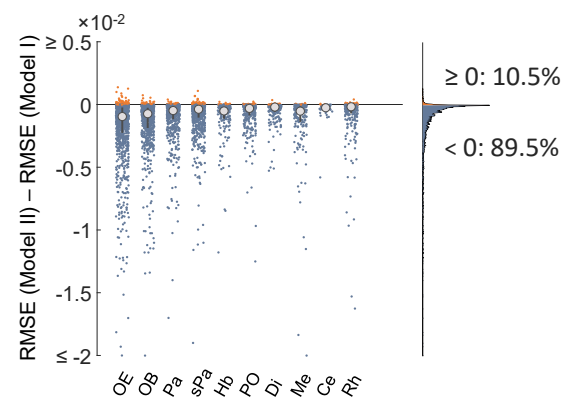

D

Model III (without interaction regressor)

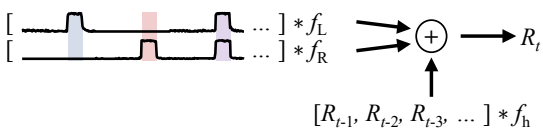

Model IV (with interaction regressor)

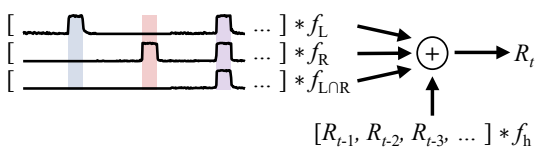

F

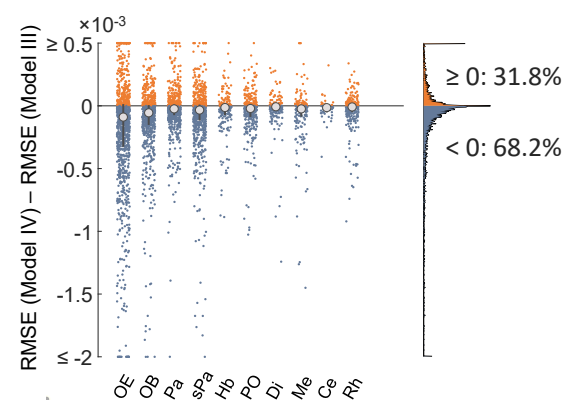

E
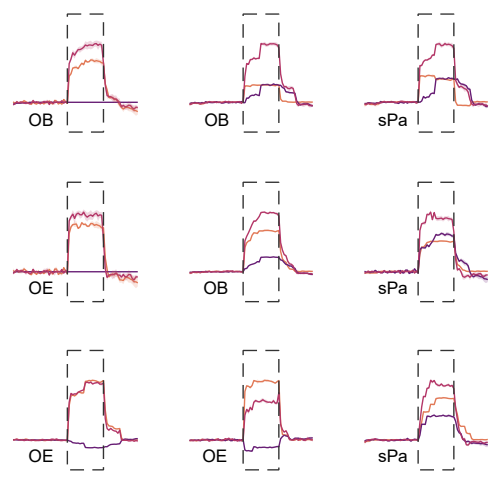

— b-STIM

Ipsi-STIM
Contra-STIM

Predicted responses (Model IV)
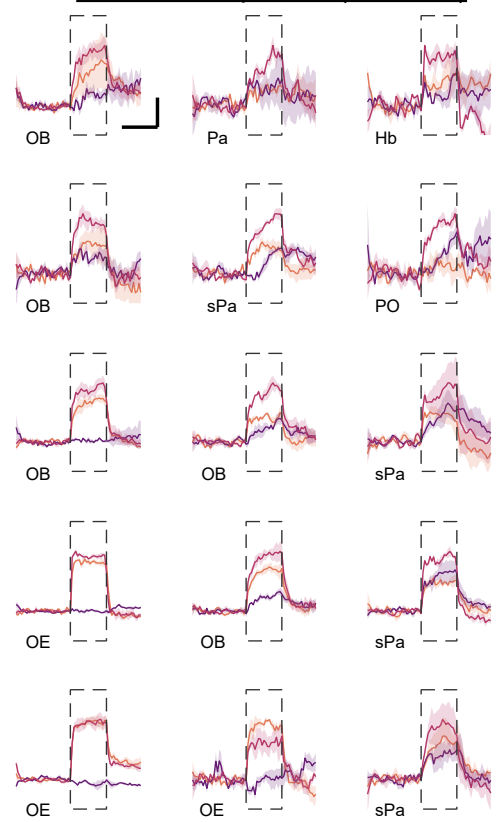

Supplementary Figure 7. Generalized linear model (GLM) fitting to neuronal responses. (A) Two GLMs using only stimulus regressors for the individual OPs (Model I) or with the addition of a regressor to account for input interactions (Model II). $f_{\mathrm{L}}, f_{R}$ and $f_{\mathrm{L} \cap \mathrm{R}}$ represent kernels for convolving with the 1-STIM, r-STIM stimulus profiles and interaction regressors fitted to the models, respectively. Colored shadows indicate the chemical stimulus delivery windows. (B) Example trial-averaged predicted responses to ipsilateral (ipsi-STIM, orange), contralateral (contra-STIM, violet) or bilateral (b-STIM, cherry) OP stimulation of the same set of ROIs shown in Figure 6D with Model II (i.e., with interaction regressor). Shadow shows the SEM for each trace. (C) The distribution of root-mean-square error (RMSE) difference between Model II and Model I for individual ROIs in the different brain regions ( $n=9$ larvae). Large dots, upper and lower limits of lines: medians, 75 and 25 percentiles, respectively. Right panel: The distribution of RMSE difference pooled across regions. The proportions of ROI with RMSE(Model II) - RMSE(Model I) $\geq 0$ (favoring Model I) and RMSE(Model II) - RMSE(Model I) $<0$ (favoring Model II) are indicated. (D), (E) and (F): Similar to (A) - (C), but Model III and Model IV both have an additional regressor corresponding to the activity history of each ROI to be convolved with its fitted activity history kernel $\left(f_{\mathrm{h}}\right)$. Example trial-averaged predicted responses of the same set of ROIs shown in Figure 6D with Model IV are shown in (E). Dashed rectangle in (B) and (E) indicates stimulus window. Scale bars in (B) and (E): 10 seconds (horizontal) and 0.5 normalized $d F / F$ (vertical). 

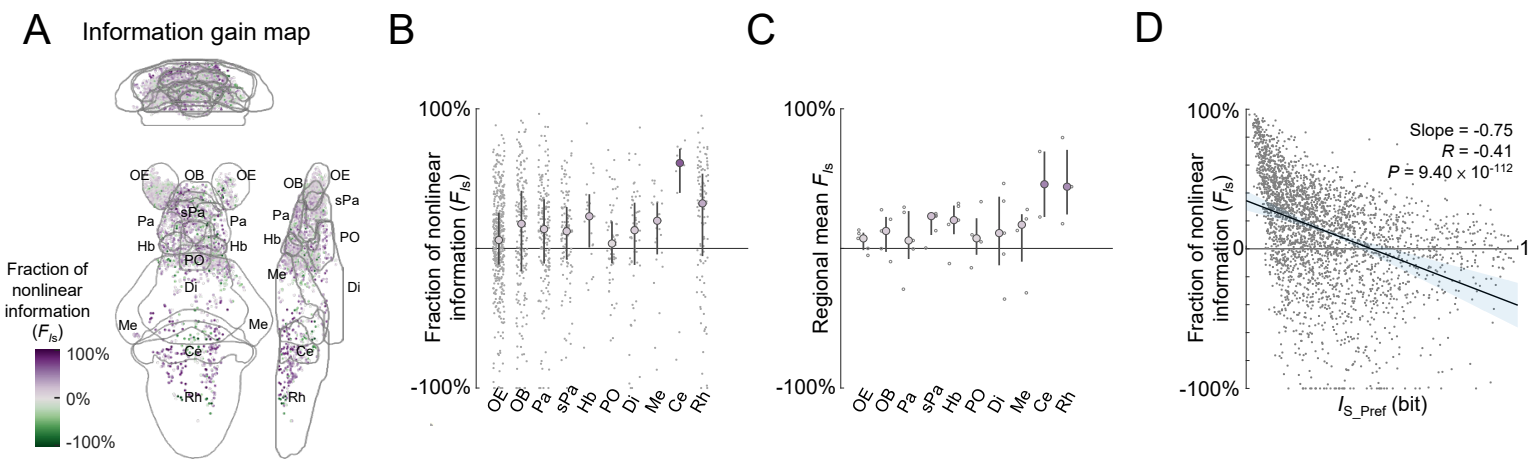

E
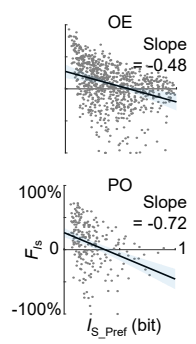$$
\text { . }
$$ 